\title{
Abelian covers of surfaces and the homology of the level $L$ mapping class group
}

\author{
Andrew Putman* \\ October 29, 2018
}

\begin{abstract}
We calculate the first homology group of the mapping class group with coefficients in the first rational homology group of the universal abelian $\mathbb{Z} / L$-cover of the surface. If the surface has one marked point, then the answer is $\mathbb{Q}^{\tau(L)}$, where $\tau(L)$ is the number of positive divisors of $L$. If the surface instead has one boundary component, then the answer is $\mathbb{Q}$. We also perform the same calculation for the level $L$ subgroup of the mapping class group. Set $H_{L}=\mathrm{H}_{1}\left(\Sigma_{g} ; \mathbb{Z} / L\right)$. If the surface has one marked point, then the answer is $\mathbb{Q}\left[H_{L}\right]$, the rational group ring of $H_{L}$. If the surface instead has one boundary component, then the answer is $\mathbb{Q}$.
\end{abstract}

\section{Introduction}

Let $\Sigma_{g, b}^{n}$ be an oriented genus $g$ surface with $b$ boundary components and $n$ marked points and let $\operatorname{Mod}_{g, b}^{n}$ be its mapping class group. This is the group of homotopy classes of orientation-preserving diffeomorphisms of $\Sigma_{g, b}^{n}$ that act as the identity on the boundary components and marked points. We will usually omit the $b$ and the $n$ if they vanish. The homology groups of $\operatorname{Mod}_{g, b}^{n}$, which play important roles in both algebraic geometry and low-dimensional topology, have been studied intensely for the past 40 years. The culmination of much recent work is the resolution of the Mumford conjecture by Madsen and Weiss [17], which identifies $\mathrm{H}^{*}\left(\operatorname{Mod}_{g, b}^{n} ; \mathbb{Q}\right)$ in a stable range.

Twisted coefficient systems. For many applications, it is important to know the homology groups of $\operatorname{Mod}_{g, b}^{n}$ with respect to various twisted coefficient systems. For simplicity, assume that $(b, n) \in\{(0,0),(1,0),(0,1)\}$. A lot is known about coefficient systems that factor through the standard symplectic representation of $\operatorname{Mod}_{g, b}^{n}$. This is the natural representation $\operatorname{Mod}_{g, b}^{n} \rightarrow \mathrm{Sp}_{2 g}(\mathbb{Z})$ that arises from the action of $\operatorname{Mod}_{g, b}^{n}$ on $\mathrm{H}_{1}\left(\Sigma_{g, b}^{n} ; \mathbb{Z}\right)$. Its target is the symplectic group because the action preserves the algebraic intersection form. For any rational representation $V$ of the algebraic group $\mathrm{Sp}_{2 g}$, Looijenga [15] has completely determined $\mathrm{H}^{*}\left(\operatorname{Mod}_{g} ; V\right)$ as a module over $\mathrm{H}^{*}\left(\operatorname{Mod}_{g} ; \mathbb{Q}\right)$ in a stable range. Over $\mathbb{Z}$, a bit less is known. Morita [18] has calculated $\mathrm{H}_{1}\left(\operatorname{Mod}_{g, b}^{n} ; \mathrm{H}_{1}\left(\Sigma_{g, b}^{n} ; \mathbb{Z}\right)\right)$ for $g \geq 3$. For $b \geq 1$, this was later generalized by Kawazumi [14], who calculated $\mathrm{H}^{*}\left(\operatorname{Mod}_{g, b}^{n} ;\left(\mathrm{H}^{1}\left(\Sigma_{g, b}^{n} ; \mathbb{Z}\right)\right)^{\otimes k}\right)$ as a module over $\mathrm{H}^{*}\left(\operatorname{Mod}_{g, b}^{n} ; \mathbb{Z}\right)$ in a stable range.

Fix some $L \geq 2$. In this paper, we calculate the first homology group of the mapping class group with coefficients in the first rational homology group of the universal abelian $\mathbb{Z} / L$-cover of the surface (see below for the definition). We remark that this representation does not factor through $\operatorname{Sp}_{2 g}(\mathbb{Z})$. Our techniques also give results for certain finite-index subgroups of the mapping class group. These results play an important technical role in a recent pair of papers by the author $[20,21]$ that study the second cohomology group and Picard group of the moduli space of curves with level $L$ structures.

${ }^{*}$ Supported in part by NSF grant DMS-1005318 
Universal abelian $\mathbb{Z} / L$-cover. Let $K_{g}$ be the kernel of the natural map $\pi_{1}\left(\Sigma_{g}\right) \rightarrow \mathrm{H}_{1}\left(\Sigma_{g} ; \mathbb{Z} / L\right)$. The group $K_{g}$ is the fundamental group of the universal abelian $\mathbb{Z} / L$-cover of $\Sigma_{g}$. Since $\operatorname{Mod}_{g}^{1}$ fixes a basepoint on $\Sigma_{g}$, it acts on $\pi_{1}\left(\Sigma_{g}\right)$. This action preserves $K_{g}$. We thus obtain an action of $\operatorname{Mod}_{g}^{1}$ on $\mathrm{H}_{1}\left(K_{g} ; \mathbb{Q}\right)$, the first homology group of the universal abelian $\mathbb{Z} / L$-cover of $\Sigma_{g}$. This representation has been previously studied by Looijenga [16], who essentially determined its image.

Remark. In [16], Looijenga more generally studied the actions of appropriate finite-index subgroups of $\operatorname{Mod}_{g}^{1}$ on the first rational homology groups $V$ of arbitrary finite abelian covers of $\Sigma_{g}$. Letting $\operatorname{Mod}_{g}^{1}(L)$ denote the level $L$ subgroup of $\operatorname{Mod}_{g}^{1}$ (see below), we can choose $L$ so that $\operatorname{Mod}_{g}^{1}(L)$ acts on $V$. It then follows from Lemma 2.2 below that $V$ appears a direct summand in the $\operatorname{Mod}_{g}^{1}(L)$-module $\mathrm{H}_{1}\left(K_{g} ; \mathbb{Q}\right)$, so one can use our results to study $V$ as well.

Statements of theorems. Let $\tau(L)$ be the number of positive divisors of $L$ (including 1 and $L$ ). Our first theorem is as follows.

Theorem A. For $g \geq 4$ and $L \geq 2$, we have $\mathrm{H}_{1}\left(\operatorname{Mod}_{g}^{1} ; \mathrm{H}_{1}\left(K_{g} ; \mathbb{Q}\right)\right) \cong \mathbb{Q}^{\tau(L)}$.

In fact, our proof of Theorem A also gives a result for the level L subgroup of $\operatorname{Mod}_{g}^{1}$, denoted $\operatorname{Mod}_{g}^{1}(L)$. This is the kernel of the action of $\operatorname{Mod}_{g}^{1}$ on $\mathrm{H}_{1}\left(\Sigma_{g}^{1} ; \mathbb{Z} / L\right)$. Far less is known about its homology. The only previous result of which the author is aware is a paper of Hain [9] that calculates $\mathrm{H}_{1}\left(\operatorname{Mod}_{g, b}^{n}(L) ; V\right)$ for rational representations $V$ of the algebraic group $\mathrm{Sp}_{2 g}$. Our theorem is as follows.

Theorem B. For $g \geq 4$ and $L \geq 2$, we have $\mathrm{H}_{1}\left(\operatorname{Mod}_{g}^{1}(L) ; \mathrm{H}_{1}\left(K_{g} ; \mathbb{Q}\right)\right) \cong \mathbb{Q}\left[H_{L}\right]$, where $H_{L}$ equals $\mathrm{H}_{1}\left(\Sigma_{g} ; \mathbb{Z} / L\right)$ and $\mathbb{Q}\left[H_{L}\right]$ is the rational group ring of the abelian group $H_{L}$.

Remark. Both $\mathrm{H}_{1}\left(\operatorname{Mod}_{g}^{1}(L) ; \mathrm{H}_{1}\left(K_{g} ; \mathbb{Q}\right)\right)$ and $\mathbb{Q}\left[H_{L}\right]$ possess natural $\operatorname{Mod}_{g}^{1}$-actions. The action of $\operatorname{Mod}_{g}^{1}$ on $\mathrm{H}_{1}\left(\operatorname{Mod}_{g}^{1}(L) ; \mathrm{H}_{1}\left(K_{g} ; \mathbb{Q}\right)\right)$ comes from conjugation, and the action on $\mathbb{Q}\left[H_{L}\right]$ factors through the symplectic group. The isomorphism in Theorem B is equivariant with respect to these actions.

Somewhat surprisingly, things are quite different for surfaces with boundary. Define $\operatorname{Mod}_{g, 1}(L)$ to be the kernel of the action of $\operatorname{Mod}_{g, 1}$ on $\mathrm{H}_{1}\left(\Sigma_{g, 1} ; \mathbb{Z} / L\right)$. Fixing a basepoint for $\pi_{1}\left(\Sigma_{g, 1}\right)$ on $\partial \Sigma_{g, 1}$, the groups $\operatorname{Mod}_{g, 1}$ and $\operatorname{Mod}_{g, 1}(L)$ act on $\pi_{1}\left(\Sigma_{g, 1}\right)$. Define $K_{g, 1}$ to be the kernel of the map $\pi_{1}\left(\Sigma_{g, 1}\right) \rightarrow \mathrm{H}_{1}\left(\Sigma_{g, 1} ; \mathbb{Z} / L\right)$. The group $K_{g, 1}$ is the fundamental group of the universal abelian $\mathbb{Z} / L$-cover of $\Sigma_{g, 1}$ and is preserved by the actions of $\operatorname{Mod}_{g, 1}$ and $\operatorname{Mod}_{g, 1}(L)$. We then have the following theorem.

Theorem C. For $g \geq 4$ and $L \geq 2$, we have

$$
\mathrm{H}_{1}\left(\operatorname{Mod}_{g, 1} ; \mathrm{H}_{1}\left(K_{g, 1} ; \mathbb{Q}\right)\right) \cong \mathrm{H}_{1}\left(\operatorname{Mod}_{g, 1}(L) ; \mathrm{H}_{1}\left(K_{g, 1} ; \mathbb{Q}\right)\right) \cong \mathbb{Q}
$$

Remark. The group $\operatorname{Mod}_{g}$ does not act on the universal abelian $\mathbb{Z} / L$-cover of $\Sigma_{g}$. Each individual mapping class can be lifted to a diffeomorphism of the cover, but a fixed basepoint is necessary to make this lift canonical and thereby provide a representation of the entire group. The best one can achieve is as follows. There is a Birman exact sequence (see $\S 2.3$ ) of the form

$$
1 \longrightarrow \pi_{1}\left(\Sigma_{g}\right) \longrightarrow \operatorname{Mod}_{g}^{1} \longrightarrow \operatorname{Mod}_{g} \longrightarrow 1
$$

Since $\operatorname{Mod}_{g}^{1}$ acts on $\mathrm{H}_{1}\left(K_{g} ; \mathbb{Q}\right)$, the group $\operatorname{Mod}_{g}$ acts on the $\left(\mathrm{H}_{1}\left(K_{g} ; \mathbb{Q}\right)\right)_{\pi_{1}\left(\Sigma_{g}\right)}$. However, using the transfer map (see Lemma 2.3 below) one can show that this ring of coinvariants is simply $\mathrm{H}_{1}\left(\Sigma_{g} ; \mathbb{Q}\right)$, so no new representation is obtained.

Comments on the proofs. The key observation underlying the proofs of our theorems is as follows. The group $\operatorname{Mod}_{g}^{1}$ contains a natural copy of $\pi_{1}\left(\Sigma_{g}\right)$, known as the "point-pushing subgroup" (see $\S 2.3$ below). This fits into the Birman exact sequence (1) above. While the action of $\operatorname{Mod}_{g}^{1}$ on $K_{g}$ is very complicated, the 
action of $\pi_{1}\left(\Sigma_{g}\right)$ on $K_{g} \triangleleft \pi_{1}\left(\Sigma_{g}\right)$ is simply conjugation. Moreover, it turns out that in some vague sense the action of $\operatorname{Mod}_{g}^{1}$ on $\mathrm{H}_{1}\left(K_{g} ; \mathbb{Q}\right)$ is "concentrated" in the action of $\pi_{1}\left(\Sigma_{g}\right)$ on $\mathrm{H}_{1}\left(K_{g} ; \mathbb{Q}\right)$. Intuitively, this happens because (as noted in the remark above) the quotient of $\operatorname{Mod}_{g}^{1}$ by $\pi_{1}\left(\Sigma_{g}\right)$, namely $\operatorname{Mod}_{g}$, does not act in any natural way on $K_{g}$. We prove our theorems by carefully examining all of these groups and actions.

Some related results. Some additional related results should be mentioned. First, Ivanov [12] has proven a homological stability result for the homology of $\operatorname{Mod}_{g, 1}$ with respect to very general systems of coefficients (those of "bounded degree"; the system $\mathrm{H}_{1}\left(K_{g, 1} ; \mathbb{Z}\right)$ satisfies this condition). This generalizes Harer's [10] well-known untwisted homological stability theorem for the mapping class group. Ivanov's theorem has been extended to $\Sigma_{g, b}$ for $b>1$ by Boldsen [5]. We remark that such a result is false for closed surfaces. Indeed, in [18, Corollary 5.4], Morita showed that

$$
\mathrm{H}_{1}\left(\operatorname{Mod}_{g} ; \mathrm{H}_{1}\left(\Sigma_{g} ; \mathbb{Z}\right)\right) \cong \mathbb{Z} /(2 g-2) \mathbb{Z}
$$

for $g \geq 2$. In a somewhat different direction, a recent series of papers by Anderson and Villemoes $[1,2,3]$ calculate the first homology groups of $\operatorname{Mod}_{g, b}^{n}$ with coefficients in certain spaces of functions on representations varieties of $\operatorname{Mod}_{g, b}^{n}$.

Outline of paper. In $\S 2$, we discuss some background results about group cohomology and the mapping class group. Next, in $\S 3$ we introduce a number of groups and group actions that will play important roles in our paper. At the end of this section, we state two key technical lemmas whose proofs are postponed until later. In $\S 4$, we prove our main theorems (assuming the truth of these two technical lemmas). In $\S 5$, we give the outline of the proof of our two key technical lemmas, reducing them to two other results, the first of which is proven in $\S 7$ (using some preliminary calculations that are first done in $\S 6$ ) and the second in $\S 8$.

Notation and conventions. We will denote by $i(x, y) \in \mathbb{Z} / L$ the algebraic intersection number of $x, y \in$ $\mathrm{H}_{1}\left(\Sigma_{g} ; \mathbb{Z} / L\right)$. All surfaces we mention will contain a basepoint unless otherwise specified, and all maps between surfaces will respect this basepoint. Also, if $G$ is a group, then we define $\left[g_{1}, g_{2}\right]=g_{1} g_{2} g_{1}^{-1} g_{2}^{-1}$ and $g_{1}^{g_{2}}=g_{2} g_{1} g_{2}^{-1}$ for $g_{1}, g_{2} \in G$.

Acknowledgments. I wish to thank an anonymous referee who pointed out Lemma 3.2 below to me. This dramatically simplified my original proofs.

\section{Preliminaries}

\subsection{Group homology}

We begin by reviewing some facts about group homology and establishing some notation (see [6] for more details).

Degree zero. Let $G$ be a group and $M$ be a $G$-module. The coinvariants of $M$, denoted $M_{G}$, is the quotient $M / K$, where $K$ is the submodule spanned by the set $\{g \cdot x-x \mid x \in M, g \in G\}$. We have $\mathrm{H}_{0}(G ; M)=M_{G}$.

The five-term exact sequence. Let

$$
1 \longrightarrow K \longrightarrow G \longrightarrow Q \longrightarrow 1
$$

be a short exact sequence of groups and let $M$ be a $G$-module. We then have a 5 -term exact sequence

$$
\mathrm{H}_{2}(G ; M) \longrightarrow \mathrm{H}_{2}\left(Q ; M_{K}\right) \longrightarrow\left(\mathrm{H}_{1}(K ; M)\right)_{Q} \longrightarrow \mathrm{H}_{1}(G ; M) \longrightarrow \mathrm{H}_{1}\left(Q ; M_{K}\right) \longrightarrow 0 .
$$


The long exact sequence. Let $G$ be a group and let

$$
0 \longrightarrow M_{1} \longrightarrow M_{2} \longrightarrow M_{3} \longrightarrow 0
$$

be a short exact sequence of $G$-modules. Then there is a long exact sequence of the form

$$
\cdots \longrightarrow \mathrm{H}_{k}\left(G ; M_{1}\right) \longrightarrow \mathrm{H}_{k}\left(G ; M_{2}\right) \longrightarrow \mathrm{H}_{k}\left(G ; M_{3}\right) \longrightarrow \mathrm{H}_{k-1}\left(G ; M_{1}\right) \longrightarrow \cdots
$$

The transfer map. If $G_{2}<G_{1}$ are groups satisfying $\left[G_{1}: G_{2}\right]<\infty$ and $M$ is a $G_{1}$-module, then for all $k$ there exists a transfer map of the form $t: \mathrm{H}_{k}\left(G_{1} ; M\right) \rightarrow \mathrm{H}_{k}\left(G_{2} ; M\right)$ (see, e.g., [6, Chapter III.9]). The key property of $t$ (see [6, Proposition III.9.5]) is that if $i: \mathrm{H}_{k}\left(G_{2} ; M\right) \rightarrow \mathrm{H}_{k}\left(G_{1} ; M\right)$ is the map induced by the inclusion, then $i \circ t: \mathrm{H}_{k}\left(G_{1} ; M\right) \rightarrow \mathrm{H}_{k}\left(G_{1} ; M\right)$ is multiplication by $\left[G_{1}: G_{2}\right]$. In particular, if $M$ is a $G_{1}$-vector space over $\mathbb{Q}$, then we obtain a right inverse $\frac{1}{\left[G_{1}: G_{2}\right]} t$ to $i$. This yields the following standard lemma.

Lemma 2.1. Let $G_{2}<G_{1}$ be groups satisfying $\left[G_{1}: G_{2}\right]<\infty$ and let $M$ be a $G_{1}$-vector space over $\mathbb{Q}$. Then the map $\mathrm{H}_{k}\left(G_{2} ; M\right) \rightarrow \mathrm{H}_{k}\left(G_{1} ; M\right)$ is surjective for all $k \geq 0$.

Assume now that $M=\mathbb{Q}$ and that $\Gamma$ is a group acting on $G_{2}$ and $G_{1}$ such that the inclusion is $\Gamma$-equivariant. The induced map $\mathrm{H}_{k}\left(G_{2} ; M\right) \rightarrow \mathrm{H}_{k}\left(G_{1} ; M\right)$ is therefore $\Gamma$-equivariant. Moreover, the map $t: \mathrm{H}_{k}\left(G_{1} ; M\right) \rightarrow$ $\mathrm{H}_{k}\left(G_{2} ; M\right)$ is also $\Gamma$-equivariant, so the surjection $\mathrm{H}_{k}\left(G_{2} ; M\right) \rightarrow \mathrm{H}_{k}\left(G_{1} ; M\right)$ splits in a $\Gamma$-equivariant manner. We obtain the following lemma.

Lemma 2.2. Fix $k \geq 0$, and let $G_{2}<G_{1}$ be groups satisfying $\left[G_{1}: G_{2}\right]<\infty$. Let $\Gamma$ be a group acting on $G_{1}$ and $G_{2}$ such that the inclusion map $G_{2} \rightarrow G_{1}$ is $\Gamma$-equivariant. Define $C$ to be the kernel of the surjection $\mathrm{H}_{k}\left(G_{2} ; \mathbb{Q}\right) \rightarrow \mathrm{H}_{k}\left(G_{1} ; \mathbb{Q}\right)$. We then have a $\Gamma$-invariant splitting $\mathrm{H}_{k}\left(G_{2} ; \mathbb{Q}\right) \cong \mathrm{H}_{k}\left(G_{1} ; \mathbb{Q}\right) \oplus C$.

Finally, for finite-index normal subgroups, the Hochschild-Serre spectral sequences implies the following strengthening of Lemma 2.1.

Lemma 2.3. Let $G_{2} \triangleleft G_{1}$ be groups satisfying $\left[G_{1}: G_{2}\right]<\infty$ and let $M$ be a $G_{1}$-vector space over $\mathbb{Q}$. Then $\mathrm{H}_{k}\left(G_{1} ; M\right) \cong\left(\mathrm{H}_{k}\left(G_{2} ; M\right)\right)_{G_{1}}$ for all $k \geq 0$.

\subsection{Rational group rings}

Let $G$ be a finite group and let $\mathbb{Q}[G]$ be the rational group ring of $G$. We will consider $\mathbb{Q}[G]$ to be a left $G$-module. Let $\varepsilon: \mathbb{Q}[G] \rightarrow \mathbb{Q}$ be the augmentation map, i.e. the unique linear map such that $\varepsilon(g)=1$ for all $g \in G$. The map $\varepsilon$ is a map of $G$-modules, where $\mathbb{Q}$ has the trivial $G$-action. Its kernel is the augmentation ideal $I(G)$. We thus have a short exact sequence of $G$-modules

$$
0 \longrightarrow I(G) \longrightarrow \mathbb{Q}[G] \stackrel{\varepsilon}{\longrightarrow} \mathbb{Q} \longrightarrow 0 .
$$

Set $\theta=\sum_{g \in G} g \in \mathbb{Q}[G]$. The element $\theta$ is invariant under $G$, and the exact sequence (2) splits via the $G$ equivariant map $\psi: \mathbb{Q} \rightarrow \mathbb{Q}[G]$ defined by $\psi(1)=\frac{1}{|G|} \theta$. The associated projection $\phi: \mathbb{Q}[G] \rightarrow I(G)$ satisfies $\operatorname{ker}(\phi)=\langle\theta\rangle$. From these considerations, we obtain the following lemma.

Lemma 2.4. Let $G$ be a finite group. Then $\mathbb{Q}[G] \cong \mathbb{Q} \oplus I(G)$, where $I(G)$ is isomorphic to the quotient of $\mathbb{Q}[G]$ by $\langle\theta\rangle$.

\subsection{The mapping class group}

Dehn twists. We will denote by $T_{\gamma}$ the left Dehn twist about a simple closed curve $\gamma$ on a surface. 

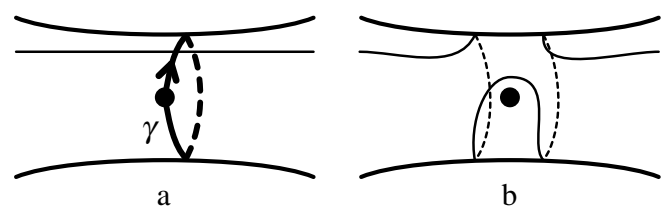

Figure 1: a. $\gamma \in \pi_{1}\left(\Sigma_{g, b}^{p}, *\right)$ can be realized by a simple closed curve. c. $\rho_{\gamma}=T_{\gamma_{1}} T_{\gamma_{2}}^{-1}$

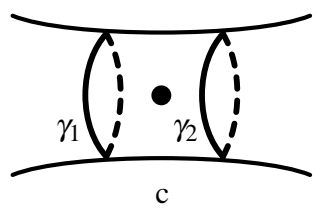

b. The effect of the "point-pushing" map $\rho_{\gamma}$.

The Birman exact sequence. For simplicity, assume that $g \geq 2$. The Birman exact sequence describes the effect on the mapping class group of deleting a marked point or gluing a disc to a boundary component. The first version, due to Birman (see [4]), is of the form

$$
1 \longrightarrow \pi_{1}\left(\Sigma_{g, b}^{p}, *\right) \longrightarrow \operatorname{Mod}_{g, b}^{p+1} \longrightarrow \operatorname{Mod}_{g, b}^{p} \longrightarrow 1 .
$$

Here $*$ is a marked point and the $\operatorname{map}_{\operatorname{Mod}_{g, b}}^{p+1} \rightarrow \operatorname{Mod}_{g, b}^{p}$ comes from deleting $*$. For $\gamma \in \pi_{1}\left(\Sigma_{g, b}^{p}, *\right)$, the associated mapping class in the kernel of (3) "pushes" the deleted marked point around the path $\gamma$. For this reason, the kernel $\pi_{1}\left(\Sigma_{g, b}^{p}, *\right)$ is known as the "point-pushing subgroup". If $\gamma \in \pi_{1}\left(\Sigma_{g, b}^{p}, *\right)$ can be realized by a simple closed curve, then there is a nice formula for the associated "point-pushing" mapping class $\rho_{\gamma}$. Namely, let $\gamma_{1}$ and $\gamma_{2}$ be the boundary components of a regular neighborhood of $\gamma$ (see Figure 1.c). Assume that $\gamma_{1}$ lies to the left of $\gamma$ and $\gamma_{2}$ to the right. Then as is clear from Figure 1.a-b, we have $\rho_{\gamma}=T_{\gamma_{1}} T_{\gamma_{2}}^{-1}$.

The second form of the Birman exact sequence, due to Johnson [13], is of the form

$$
1 \longrightarrow \pi_{1}\left(U \Sigma_{g, b}^{p}\right) \longrightarrow \operatorname{Mod}_{g, b+1}^{p} \longrightarrow \operatorname{Mod}_{g, b}^{p} \longrightarrow 1 .
$$

Here $U \Sigma_{g, b}^{p}$ is the unit tangent bundle of $\Sigma_{g, b}^{p}$ and the $\operatorname{map}_{\operatorname{Mod}_{g, b+1}}^{p} \rightarrow \operatorname{Mod}_{g, b}^{p}$ comes from gluing a disc to a boundary component $\beta$ of $\Sigma_{g, b+1}^{p}$ and extending mapping classes by the identity. The fiber of the kernel $\pi_{1}\left(U \Sigma_{g, b}^{p}\right)$ corresponds to the mapping class $T_{\beta}$.

The group $\operatorname{Mod}_{g, b+1}^{p}$ acts on $\mathrm{H}_{1}\left(\Sigma_{g, b+1}^{p} ; \mathbb{Q}\right)$. Restrict this action to $\pi_{1}\left(U \Sigma_{g, b}^{p}\right)<\operatorname{Mod}_{g, b+1}^{p}$. Since $T_{\beta}$ acts trivially on $\mathrm{H}_{1}\left(\Sigma_{g, b+1}^{p} ; \mathbb{Q}\right)$, the action of $\pi_{1}\left(U \Sigma_{g, b}^{p}\right)$ on $\mathrm{H}_{1}\left(\Sigma_{g, b+1}^{p} ; \mathbb{Q}\right)$ factors through an action of $\pi_{1}\left(\Sigma_{g, b}^{p}, *\right)$. This action has the following simple description.

Lemma 2.5. The above action of $\pi_{1}\left(\Sigma_{g, b}^{p}, *\right)$ on $\mathrm{H}_{1}\left(\Sigma_{g, b+1}^{p} ; \mathbb{Q}\right)$ is given by the formula

$$
\gamma(v)=v+i([\gamma], v) \cdot[\beta] \quad\left(\gamma \in \pi_{1}\left(\Sigma_{g, b}^{p}, *\right), v \in \mathrm{H}_{1}\left(\Sigma_{g, b+1}^{p} ; \mathbb{Q}\right)\right),
$$

where the boundary component $\beta$ is oriented such that the interior of the surface is to its right.

Proof. It is enough to check this for elements $\gamma \in \pi_{1}\left(\Sigma_{g, b}^{p}, *\right)$ that can be realized by simple closed curves. For such curves, this formula is immediate from Figure 1.a-b.

The level $L$ subgroup. Now assume that $b=p=0$ and fix some $L \geq 2$. The kernels of (3) and (4) both lie in the level $L$ subgroup of the mapping class group. We thus have short exact sequences

$$
1 \longrightarrow \pi_{1}\left(\Sigma_{g}\right) \longrightarrow \operatorname{Mod}_{g}^{1}(L) \longrightarrow \operatorname{Mod}_{g}(L) \longrightarrow 1
$$

and

$$
1 \longrightarrow \pi_{1}\left(U \Sigma_{g}\right) \longrightarrow \operatorname{Mod}_{g, 1}(L) \longrightarrow \operatorname{Mod}_{g}(L) \longrightarrow 1 .
$$

We will also refer to these as Birman exact sequences.

We will need two cohomological results about the level $L$ subgroups of the mapping class group, both of which are due to Hain. To simplify their statements, we will denote the whole mapping class group by $\operatorname{Mod}_{g, 1}(1)$ and $\operatorname{Mod}_{g}^{1}(1)$. 
Theorem 2.6 (Hain, [9]). For $g \geq 3$ and $L \geq 1$, we have $\mathrm{H}_{1}\left(\operatorname{Mod}_{g, 1}(L) ; \mathbb{Q}\right)=0$.

Theorem 2.7 (Hain, [9]). For $g \geq 3$ and $L \geq 1$, we have

$$
\mathrm{H}_{1}\left(\operatorname{Mod}_{g, 1}(L) ; \mathrm{H}_{1}\left(\Sigma_{g} ; \mathbb{Q}\right)\right) \cong \mathrm{H}_{1}\left(\operatorname{Mod}_{g}^{1}(L) ; \mathrm{H}_{1}\left(\Sigma_{g} ; \mathbb{Q}\right)\right) \cong \mathbb{Q} .
$$

Remark. In fact, in [9] Hain calculated $\mathrm{H}_{1}\left(\operatorname{Mod}_{g, b}^{p}(L) ; M\right)$ for all rational representations $M$ of the algebraic group $\mathrm{Sp}_{2 g}$.

\section{The cast of characters}

\subsection{Homology groups of abelian covers}

As in the introduction, define

$$
K_{g}=\operatorname{ker}\left(\pi_{1}\left(\Sigma_{g}\right) \rightarrow \mathrm{H}_{1}\left(\Sigma_{g} ; \mathbb{Z} / L\right)\right) \quad \text { and } \quad K_{g, 1}=\operatorname{ker}\left(\pi_{1}\left(\Sigma_{g, 1}\right) \rightarrow \mathrm{H}_{1}\left(\Sigma_{g, 1} ; \mathbb{Z} / L\right)\right) .
$$

Here the basepoint for $\pi_{1}\left(\Sigma_{g, 1}\right)$ lies on $\partial \Sigma_{g, 1}$. Also, let $S_{g}^{K}$ (resp. $\left.S_{g, 1}^{K}\right)$ be the cover of $\Sigma_{g}$ (resp. $\left.\Sigma_{g, 1}\right)$ corresponding to $K_{g}$ (resp. $\left.K_{g, 1}\right)$. We will identify $\mathrm{H}_{1}\left(K_{g} ; \mathbb{Q}\right)$ and $\mathrm{H}_{1}\left(K_{g, 1} ; \mathbb{Q}\right)$ with $\mathrm{H}_{1}\left(S_{g}^{K} ; \mathbb{Q}\right)$ and $\mathrm{H}_{1}\left(S_{g, 1}^{K} ; \mathbb{Q}\right)$, respectively.

We have actions of $\operatorname{Mod}_{g}^{1}$ and $\operatorname{Mod}_{g, 1}$ on $K_{g}$ and $K_{g, 1}$, respectively. Define

$$
C_{g}=\operatorname{ker}\left(\mathrm{H}_{1}\left(K_{g} ; \mathbb{Q}\right) \rightarrow \mathrm{H}_{1}\left(\Sigma_{g} ; \mathbb{Q}\right)\right) \quad \text { and } \quad C_{g, 1}=\operatorname{ker}\left(\mathrm{H}_{1}\left(K_{g, 1} ; \mathbb{Q}\right) \rightarrow \mathrm{H}_{1}\left(\Sigma_{g, 1} ; \mathbb{Q}\right)\right) .
$$

By Lemma 2.2, we have mapping class group invariant decompositions

$$
\mathrm{H}_{1}\left(K_{g} ; \mathbb{Q}\right) \cong \mathrm{H}_{1}\left(\Sigma_{g} ; \mathbb{Q}\right) \oplus C_{g} \quad \text { and } \quad \mathrm{H}_{1}\left(K_{g, 1} ; \mathbb{Q}\right) \cong \mathrm{H}_{1}\left(\Sigma_{g, 1} ; \mathbb{Q}\right) \oplus C_{g, 1} .
$$

The surjective map $\pi_{1}\left(\Sigma_{g, 1}\right) \rightarrow \pi_{1}\left(\Sigma_{g}\right)$ induced by gluing a disc to the boundary component of $\Sigma_{g, 1}$ restricts to a surjection $K_{g, 1} \rightarrow K_{g}$. Let $I_{g}$ be its kernel, so we have a short exact sequence

$$
0 \longrightarrow I_{g} \longrightarrow K_{g, 1} \longrightarrow K_{g} \longrightarrow 0 .
$$

We now prove the following.

Lemma 3.1. For $g \geq 1$ and $L \geq 2$, the vector space $I_{g}$ is isomorphic as a $\operatorname{Mod}_{g, 1}$-module to the augmentation ideal of $\mathbb{Q}\left[H_{L}\right]$, where $H_{L}=\mathrm{H}_{1}\left(\Sigma_{g, 1} ; \mathbb{Z} / L\right)$.

Proof. Let $* \in \partial \Sigma_{g, 1}$ be the basepoint and $\tilde{*} \in S_{g, 1}^{K}$ be a lift of $*$, so $K_{g, 1}=\pi_{1}\left(S_{g, 1}^{K}, \tilde{*}\right)$. The subgroup $I_{g}<$ $\mathrm{H}_{1}\left(S_{g, 1}^{K} ; \mathbb{Q}\right)$ is exactly the subgroup generated by the homology classes of the boundary components of $S_{g, 1}^{K}$. The group of deck transformations $H_{L}$ acts on these boundary components. Let $\gamma \in \pi_{1}\left(\Sigma_{g, 1}, *\right)$ be the simple closed curve that goes once around the boundary component with the surface to its right. Since $\gamma$ lifts to a simple closed curve $\tilde{\gamma} \in \pi_{1}\left(S_{g, 1}^{K}, \tilde{*}\right)$, the action of $H_{L}$ on the boundary components of $\tilde{\Sigma}$ is free. For $v \in H_{L}$, let $\llbracket v \rrbracket \in I_{g}$ denote the homology class of the $v$-translate of $\tilde{\gamma}$. The only relation between the homology classes of the boundary components of a surface with boundary is that their sum is 0 . We conclude that $I_{g}$ is isomorphic to the quotient of the $\mathbb{Q}$-vector space with basis the formal symbols $\left\{\llbracket v \rrbracket \mid v \in H_{L}\right\}$ by the 1-dimensional subspace generated by $\sum_{v \in H_{L}} \llbracket v \rrbracket$. This is exactly the augmentation ideal of $\mathbb{Q}\left[H_{L}\right]$, and we are done.

Throughout the rest of this paper, we will denote by $\llbracket v \rrbracket$ the element of $I_{g}$ corresponding to $v \in H_{L}$ as in the proof above.

Remark. It is clear that $I_{g}<C_{g, 1}$, so the exact sequence (5) restricts to a short exact sequence

$$
0 \longrightarrow I_{g} \longrightarrow C_{g, 1} \longrightarrow C_{g} \longrightarrow 0 .
$$




\subsection{Actions on homology groups of abelian covers}

It is clear that $\operatorname{Mod}_{g, 1}$ acts on $K_{g, 1}$ and $\operatorname{Mod}_{g}^{1}$ acts on $K_{g}$. Letting $\beta$ be the boundary component of $\Sigma_{g, 1}$, these two mapping class groups are related by a short exact sequence

$$
1 \longrightarrow \mathbb{Z} \longrightarrow \operatorname{Mod}_{g, 1} \longrightarrow \operatorname{Mod}_{g}^{1} \longrightarrow 1,
$$

where $\mathbb{Z}$ is generated by $T_{\beta}$. Since the loop around $\beta$ lies in $K_{g, 1}$, the action of $T_{\beta}$ on $\mathrm{H}_{1}\left(K_{g, 1} ; \mathbb{Q}\right)$ is trivial. This implies that the action of $\operatorname{Mod}_{g, 1}$ on $\mathrm{H}_{1}\left(K_{g, 1} ; \mathbb{Q}\right)$ factors through an action of $\operatorname{Mod}_{g}^{1}$.

Let $\pi_{1}\left(\Sigma_{g}\right)<\operatorname{Mod}_{g}^{1}$ be the point-pushing subgroup. The action of $\operatorname{Mod}_{g}^{1}$ on $\mathrm{H}_{1}\left(K_{g} ; \mathbb{Q}\right)$ restricts to the action of $\pi_{1}\left(\Sigma_{g}\right)$ on $\mathrm{H}_{1}\left(K_{g} ; \mathbb{Q}\right)$ induced by conjugation. Restricting this action further to $K_{g}<\pi_{1}\left(\Sigma_{g}\right)$ thus yields the trivial action. However, if we instead restrict the action of $\operatorname{Mod}_{g}^{1}$ on $\mathrm{H}_{1}\left(K_{g, 1} ; \mathbb{Q}\right)$ to $K_{g}$, we do $n o t$ get a trivial action.

For $\gamma \in K_{g}$, denote by $\langle\langle\gamma\rangle\rangle$ the associated element of $\mathrm{H}_{1}\left(K_{g} ; \mathbb{Q}\right)$. Since $K_{g}<\operatorname{Mod}_{g}^{1}$ acts trivially on the kernel and cokernel of the short exact sequence

$$
0 \longrightarrow I_{g} \longrightarrow \mathrm{H}_{1}\left(K_{g, 1} ; \mathbb{Q}\right) \stackrel{\rho}{\longrightarrow} \mathrm{H}_{1}\left(K_{g} ; \mathbb{Q}\right) \longrightarrow 0,
$$

the action of $K_{g}$ on $\mathrm{H}_{1}\left(K_{g, 1} ; \mathbb{Q}\right)$ is of the form

$$
\gamma(x)=x+\omega(\langle\gamma\rangle\rangle, \rho(x)) \quad\left(\gamma \in K_{g} \text { and } x \in \mathrm{H}_{1}\left(K_{g, 1} ; \mathbb{Q}\right)\right)
$$

for some $I_{g}$-valued bilinear form $\omega(\cdot, \cdot)$ on $\mathrm{H}_{1}\left(K_{g} ; \mathbb{Q}\right)$. This bilinear form has the following nice description. Let $\langle\cdot, \cdot\rangle_{K}$ be the algebraic intersection pairing on $\mathrm{H}_{1}\left(K_{g} ; \mathbb{Q}\right)=\mathrm{H}_{1}\left(S_{g}^{K} ; \mathbb{Q}\right)$. The group $H_{L}=\mathrm{H}_{1}\left(\Sigma_{g} ; \mathbb{Z} / L\right)$ acts on $\mathrm{H}_{1}\left(S_{g}^{K} ; \mathbb{Q}\right)$ via deck transformations. The bilinear pairing in the following lemma first appeared in work of Reidemeister [22, 23] and has since been studied by many people (see, e.g., [7, 11, 16]). We will call it the Reidemeister pairing.

Lemma 3.2. For $\gamma \in K_{g}$ and $x \in K_{g}$, we have

$$
\omega(\langle\langle\gamma\rangle\rangle, x)=\sum_{v \in H_{L}}\langle v \cdot\langle\langle\gamma\rangle\rangle, x\rangle_{K} \llbracket v \rrbracket .
$$

Proof. An immediate consequence of Lemma 2.5.

\subsection{A key technical lemma}

One of the linchpins of our proofs of our main theorems is the following lemma about the action of $K_{g}$ on $C_{g, 1}<K_{g, 1}$. Its proof is lengthy and is given in $\S 5$.

Lemma 3.3. For $g \geq 4$ and $L \geq 2$, the map $\mathrm{H}_{1}\left(K_{g} ; C_{g, 1}\right) \rightarrow \mathrm{H}_{1}\left(\operatorname{Mod}_{g}^{1}(L) ; C_{g}\right)$ is the zero map.

Remark. The map $\mathrm{H}_{1}\left(K_{g} ; C_{g, 1}\right) \rightarrow \mathrm{H}_{1}\left(\operatorname{Mod}_{g}^{1}(L) ; C_{g}\right)$ factors through $\mathrm{H}_{1}\left(K_{g} ; C_{g}\right)$, and most of our hard work is devoted to characterizing the image of $\mathrm{H}_{1}\left(K_{g} ; C_{g, 1}\right)$ in $\mathrm{H}_{1}\left(K_{g} ; C_{g}\right)$. It would be much easier if we could instead prove that the map $\mathrm{H}_{1}\left(K_{g} ; C_{g}\right) \rightarrow \mathrm{H}_{1}\left(\operatorname{Mod}_{g}^{1}(L) ; C_{g}\right)$ was the zero map, but alas a careful examination of our proof of Theorem $B$ shows that this is not true.

In the course of proving Lemma 3.3, we will also prove the following.

Lemma 3.4. For $g \geq 3$ and $L \geq 2$, we have $\left(C_{g}\right)_{\pi_{1}\left(\Sigma_{g}\right)}=\left(C_{g, 1}\right)_{\pi_{1}\left(\Sigma_{g}\right)}=0$.

One useful consequence of Lemma 3.4 is the following.

Lemma 3.5. For $g \geq 3$ and $L \geq 2$, the natural map $\mathrm{H}_{1}\left(\operatorname{Mod}_{g, 1}(L) ; C_{g}\right) \rightarrow \mathrm{H}_{1}\left(\operatorname{Mod}_{g}^{1}(L) ; C_{g}\right)$ is an isomorphism. 
Proof. Let $\beta$ be the boundary component of $\Sigma_{g, 1}$. We have a short exact sequence

$$
1 \longrightarrow \mathbb{Z} \longrightarrow \operatorname{Mod}_{g, 1}(L) \longrightarrow \operatorname{Mod}_{g}^{1}(L) \longrightarrow 1
$$

where $\mathbb{Z}=\left\langle T_{\beta}\right\rangle$. The last 3 terms of the associated 5-term exact sequence with coefficients $C_{g}$ are

$$
\left(C_{g}\right)_{\operatorname{Mod}_{g}^{1}(L)} \longrightarrow \mathrm{H}_{1}\left(\operatorname{Mod}_{g, 1}(L) ; C_{g}\right) \longrightarrow \mathrm{H}_{1}\left(\operatorname{Mod}_{g}^{1}(L) ; C_{g}\right) \longrightarrow 0 .
$$

By Lemma 3.4, we have $\left(C_{g}\right)_{\operatorname{Mod}_{g}^{1}(L)}=0$, and the lemma follows.

\section{Proofs of the main theorems}

We now turn to the proofs of our main theorems. In this section, we will assume the truth of Lemmas 3.3 and 3.4 , which are proven in subsequent sections.

\subsection{Surfaces with boundary}

We begin with Theorem C, which asserts that if $g \geq 4$ and $L \geq 2$, then

$$
\mathrm{H}_{1}\left(\operatorname{Mod}_{g, 1} ; \mathrm{H}_{1}\left(K_{g, 1} ; \mathbb{Q}\right)\right) \cong \mathrm{H}_{1}\left(\operatorname{Mod}_{g, 1}(L) ; \mathrm{H}_{1}\left(K_{g, 1} ; \mathbb{Q}\right)\right) \cong \mathbb{Q}
$$

As was noted in $\S 3$, we can use Lemma 2.2 to obtain a $\operatorname{Mod}_{g, 1}$-invariant decomposition

$$
\mathrm{H}_{1}\left(K_{g, 1} ; \mathbb{Q}\right) \cong \mathrm{H}_{1}\left(\Sigma_{g, 1} ; \mathbb{Q}\right) \oplus C_{g, 1} .
$$

This implies that

$$
\mathrm{H}_{1}\left(\operatorname{Mod}_{g, 1} ; \mathrm{H}_{1}\left(K_{g, 1} ; \mathbb{Q}\right)\right) \cong \mathrm{H}_{1}\left(\operatorname{Mod}_{g, 1} ; \mathrm{H}_{1}\left(\Sigma_{g, 1} ; \mathbb{Q}\right)\right) \oplus \mathrm{H}_{1}\left(\operatorname{Mod}_{g, 1} ; C_{g, 1}\right),
$$

and similarly for $\operatorname{Mod}_{g, 1}(L)$. Theorem 2.7 says that

$$
\mathrm{H}_{1}\left(\operatorname{Mod}_{g, 1} ; \mathrm{H}_{1}\left(\Sigma_{g, 1} ; \mathbb{Q}\right)\right) \cong \mathrm{H}_{1}\left(\operatorname{Mod}_{g, 1}(L) ; \mathrm{H}_{1}\left(\Sigma_{g, 1} ; \mathbb{Q}\right)\right) \cong \mathbb{Q}
$$

To prove Theorem C, therefore, it is enough to prove the following theorem.

Theorem 4.1. For $g \geq 4$ and $L \geq 2$, we have

$$
\mathrm{H}_{1}\left(\operatorname{Mod}_{g, 1} ; C_{g, 1}\right) \cong \mathrm{H}_{1}\left(\operatorname{Mod}_{g, 1}(L) ; C_{g, 1}\right)=0 .
$$

Proof. Since $\operatorname{Mod}_{g, 1}(L)$ is a finite-index subgroup of $\operatorname{Mod}_{g, 1}$, Lemma 2.1 implies that it is enough to prove that $\mathrm{H}_{1}\left(\operatorname{Mod}_{g, 1}(L) ; C_{g, 1}\right)=0$. Associated to the Birman exact sequence

$$
1 \longrightarrow \pi_{1}\left(U \Sigma_{g}\right) \longrightarrow \operatorname{Mod}_{g, 1}(L) \longrightarrow \operatorname{Mod}_{g}(L) \longrightarrow 1
$$

is a 5 -term exact sequence in homology with coefficients in $C_{g, 1}$. The last 3 terms of this are

$$
\left(\mathrm{H}_{1}\left(\pi_{1}\left(U \Sigma_{g}\right) ; C_{g, 1}\right)\right)_{\operatorname{Mod}_{g, 1}(L)} \stackrel{f}{\longrightarrow} \mathrm{H}_{1}\left(\operatorname{Mod}_{g, 1}(L) ; C_{g, 1}\right) \longrightarrow \mathrm{H}_{1}\left(\operatorname{Mod}_{g}(L) ;\left(C_{g, 1}\right)_{\pi_{1}\left(U \Sigma_{g}\right)}\right) \longrightarrow 0 .
$$

Lemma 3.4 says that

$$
\left(C_{g, 1}\right)_{\pi_{1}\left(U \Sigma_{g}\right)}=\left(C_{g, 1}\right)_{\pi_{1}\left(\Sigma_{g}\right)}=0 .
$$

To prove the theorem, therefore, it is enough to show that $f=0$. This is equivalent to showing that the map

$$
f^{\prime}: \mathrm{H}_{1}\left(\pi_{1}\left(U \Sigma_{g}\right) ; C_{g, 1}\right) \rightarrow \mathrm{H}_{1}\left(\operatorname{Mod}_{g, 1}(L) ; C_{g, 1}\right)
$$


is the zero map.

Our goal is to deduce the fact that $f^{\prime}=0$ from Lemma 3.3. To do this, we perform a series of reductions. From the short exact sequence

$$
0 \longrightarrow I_{g} \longrightarrow C_{g, 1} \longrightarrow C_{g} \longrightarrow 0
$$

of $\operatorname{Mod}_{g, 1}(L)$-modules we obtain a long exact sequence in homology. This long exact sequence contains the segment

$$
\mathrm{H}_{1}\left(\operatorname{Mod}_{g, 1}(L) ; I_{g}\right) \longrightarrow \mathrm{H}_{1}\left(\operatorname{Mod}_{g, 1}(L) ; C_{g, 1}\right) \longrightarrow \mathrm{H}_{1}\left(\operatorname{Mod}_{g, 1}(L) ; C_{g}\right) .
$$

Since $\operatorname{Mod}_{g, 1}(L)$ acts trivially on $I_{g}$, Theorem 2.6 implies that $\mathrm{H}_{1}\left(\operatorname{Mod}_{g, 1}(L) ; I_{g}\right)=0$. It follows that the map $\mathrm{H}_{1}\left(\operatorname{Mod}_{g, 1}(L) ; C_{g, 1}\right) \rightarrow \mathrm{H}_{1}\left(\operatorname{Mod}_{g, 1}(L) ; C_{g}\right)$ is injective. Lemma 3.5 says that $\mathrm{H}_{1}\left(\operatorname{Mod}_{g, 1}(L) ; C_{g}\right) \cong$ $\mathrm{H}_{1}\left(\operatorname{Mod}_{g}^{1}(L) ; C_{g}\right)$, so we deduce that to prove that $f^{\prime}=0$, it is enough to prove that the map

$$
\mathrm{H}_{1}\left(\pi_{1}\left(U \Sigma_{g}\right) ; C_{g, 1}\right) \longrightarrow \mathrm{H}_{1}\left(\operatorname{Mod}_{g}^{1}(L) ; C_{g}\right)
$$

is the zero map. This map factors through the map

$$
\mathrm{H}_{1}\left(\pi_{1}\left(\Sigma_{g}\right) ; C_{g, 1}\right) \longrightarrow \mathrm{H}_{1}\left(\operatorname{Mod}_{g}^{1}(L) ; C_{g}\right) .
$$

Since $K_{g}$ is a finite-index subgroup of $\pi_{1}\left(\Sigma_{g}\right)$, Lemma 2.1 says that the map $\mathrm{H}_{1}\left(K_{g} ; C_{g, 1}\right) \longrightarrow \mathrm{H}_{1}\left(\pi_{1}\left(\Sigma_{g}\right) ; C_{g, 1}\right)$ is surjective. Thus to show that the map in (6) vanishes, it is enough to show that the map

$$
\mathrm{H}_{1}\left(K_{g} ; C_{g, 1}\right) \rightarrow \mathrm{H}_{1}\left(\operatorname{Mod}_{g}^{1}(L) ; C_{g}\right)
$$

vanishes, which is exactly the content of Lemma 3.3.

\subsection{Closed surfaces}

We now turn to Theorems $\mathrm{A}$ and $\mathrm{B}$, which assert that if $g \geq 4$ and $L \geq 2$, then

$$
\mathrm{H}_{1}\left(\operatorname{Mod}_{g}^{1} ; \mathrm{H}_{1}\left(K_{g} ; \mathbb{Q}\right)\right) \cong \mathbb{Q}^{\tau(L)} \quad \text { and } \quad \mathrm{H}_{1}\left(\operatorname{Mod}_{g}^{1}(L) ; \mathrm{H}_{1}\left(K_{g} ; \mathbb{Q}\right)\right) \cong \mathbb{Q}\left[H_{L}\right] .
$$

Here $\tau(L)$ is the number of positive divisors of $L$ and $H_{L} \cong \mathrm{H}_{1}\left(\Sigma_{g} ; \mathbb{Z} / L\right)$. Also, the second isomorphism should be equivariant with respect to $\operatorname{Mod}_{g}^{1}$ actions on $\mathrm{H}_{1}\left(\operatorname{Mod}_{g}^{1}(L) ; \mathrm{H}_{1}\left(K_{g} ; \mathbb{Q}\right)\right)$ and $\mathbb{Q}\left[H_{L}\right]$. We begin by deriving Theorem A from Theorem B.

Proof of Theorem A, assuming Theorem B. Lemma 2.3 implies that

$$
\mathrm{H}_{1}\left(\operatorname{Mod}_{g}^{1} ; \mathrm{H}_{1}\left(K_{g} ; \mathbb{Q}\right)\right) \cong\left(\mathrm{H}_{1}\left(\operatorname{Mod}_{g}^{1}(L) ; \mathrm{H}_{1}\left(K_{g} ; \mathbb{Q}\right)\right)\right)_{\operatorname{Mod}_{g}^{1}} .
$$

Applying Theorem B, we must show that $\left(\mathbb{Q}\left[H_{L}\right]\right)_{\operatorname{Mod}_{g}^{1}} \cong \mathbb{Q}^{\tau(L)}$. The vector space $\mathbb{Q}\left[H_{L}\right]$ has a basis that is permuted by the action of $\operatorname{Mod}_{g}^{1}$, namely the elements of $H_{L}$. It is enough, therefore, to show that there are $\tau(L)$ orbits of the action of $\operatorname{Mod}_{g}^{1}$ on $H_{L}$. This action factors through the surjection $\operatorname{Mod}_{g}^{1} \rightarrow \operatorname{Sp}_{2 g}(\mathbb{Z} / L)$. Let $v \in H_{L}$ be a fixed primitive vector, and set

$$
X=\{c v \mid c \text { is a positive divisor of } L\} \subset H_{L} .
$$

The set $X$ has cardinality $\tau(L)$, and clearly no two elements of $X$ are in the same $\mathrm{Sp}_{2 g}(\mathbb{Z} / L)$-orbit. Also, if $w \in H_{L}$, then there is a primitive vector $w^{\prime}$ and a positive divisor $c$ of $L$ such that $w=c w^{\prime}$. Since $\operatorname{Sp}_{2 g}(\mathbb{Z} / L)$ acts transitively on the set of primitive vectors, there is some $\phi \in \operatorname{Sp}_{2 g}(\mathbb{Z} / L)$ such that $v=\phi\left(w^{\prime}\right)$. Thus $w$ is in the same $\mathrm{Sp}_{2 g}(\mathbb{Z} / L)$-orbit as $c v \in X$. We conclude that $X$ contains a unique representative from every $\mathrm{Sp}_{2 g}(\mathbb{Z} / L)$-orbit, and we are done. 
Finally, we discuss Theorem B. Lemma 2.2 implies that there is a $\operatorname{Mod}_{g}^{1}$-invariant decomposition

$$
\mathrm{H}_{1}\left(K_{g} ; \mathbb{Q}\right) \cong \mathrm{H}_{1}\left(\Sigma_{g} ; \mathbb{Q}\right) \oplus C_{g},
$$

so we have a $\operatorname{Mod}_{g}^{1}$-invariant decomposition

$$
\mathrm{H}_{1}\left(\operatorname{Mod}_{g}^{1}(L) ; \mathrm{H}_{1}\left(K_{g} ; \mathbb{Q}\right)\right) \cong \mathrm{H}_{1}\left(\operatorname{Mod}_{g}^{1}(L) ; \mathrm{H}_{1}\left(\Sigma_{g} ; \mathbb{Q}\right)\right) \oplus \mathrm{H}_{1}\left(\operatorname{Mod}_{g}^{1}(L) ; C_{g}\right) .
$$

Also, Theorem 2.7 says that

$$
\mathrm{H}_{1}\left(\operatorname{Mod}_{g}^{1}(L) ; \mathrm{H}_{1}\left(\Sigma_{g} ; \mathbb{Q}\right)\right) \cong \mathbb{Q},
$$

so we obtain a $\operatorname{Mod}_{g}^{1}$-invariant decomposition

$$
\mathrm{H}_{1}\left(\operatorname{Mod}_{g}^{1}(L) ; \mathrm{H}_{1}\left(K_{g} ; \mathbb{Q}\right)\right) \cong \mathbb{Q} \oplus \mathrm{H}_{1}\left(\operatorname{Mod}_{g}^{1}(L) ; C_{g}\right) .
$$

By Lemma 2.4, we have $\mathbb{Q}\left[H_{L}\right] \cong \mathbb{Q} \oplus I$, where $I$ is the augmentation ideal of $\mathbb{Q}\left[H_{L}\right]$. Lemma 3.1 says that $I_{g}$ is isomorphic to the augmentation ideal of $\mathbb{Q}\left[H_{L}\right]$, so we conclude that it is enough to prove the following theorem.

Theorem 4.2. For $g \geq 4$ and $L \geq 2$, we have a $\operatorname{Mod}_{g}^{1}$-equivariant isomorphism $\mathrm{H}_{1}\left(\operatorname{Mod}_{g}^{1}(L) ; C_{g}\right) \cong I_{g}$.

Proof. Lemma 3.5 says that there is an isomorphism

$$
\mathrm{H}_{1}\left(\operatorname{Mod}_{g, 1}(L) ; C_{g}\right) \cong \mathrm{H}_{1}\left(\operatorname{Mod}_{g}^{1}(L) ; C_{g}\right) .
$$

The action of $\operatorname{Mod}_{g, 1}$ on $\mathrm{H}_{1}\left(\operatorname{Mod}_{g, 1}(L) ; C_{g}\right)$ factors through $\operatorname{Mod}_{g}^{1}$, and it is easy to see that the isomorphism in (7) is $\operatorname{Mod}_{g}^{1}$-equivariant. We deduce that it is enough to construct a $\operatorname{Mod}_{g, 1}$-equivariant isomorphism $\mathrm{H}_{1}\left(\operatorname{Mod}_{g, 1}(L) ; C_{g}\right) \cong I_{g}$. The long exact sequence in $\operatorname{Mod}_{g, 1}(L)$-homology associated to the short exact sequence

$$
0 \longrightarrow I_{g} \longrightarrow C_{g, 1} \longrightarrow C_{g} \longrightarrow 0
$$

of $\operatorname{Mod}_{g, 1}(L)$-modules contains the segment

$$
\mathrm{H}_{1}\left(\operatorname{Mod}_{g, 1}(L) ; C_{g, 1}\right) \longrightarrow \mathrm{H}_{1}\left(\operatorname{Mod}_{g, 1}(L) ; C_{g}\right) \longrightarrow I_{g} \longrightarrow\left(C_{g, 1}\right)_{\operatorname{Mod}_{g, 1}(L)} .
$$

Here we are using the fact that $\operatorname{Mod}_{g, 1}(L)$ acts trivially on $I_{g}$, so

$$
\mathrm{H}_{0}\left(\operatorname{Mod}_{g, 1}(L) ; I_{g}\right)=\left(I_{g}\right)_{\operatorname{Mod}_{g, 1}(L)}=I_{g} .
$$

Theorem 4.1 says that $\mathrm{H}_{1}\left(\operatorname{Mod}_{g, 1}(L) ; C_{g, 1}\right)=0$, and Lemma 3.4 implies that $\left(C_{g, 1}\right)_{\operatorname{Mod}_{g, 1}(L)}=0$. We obtain an isomorphism $\mathrm{H}_{1}\left(\operatorname{Mod}_{g, 1}(L) ; C_{g}\right) \cong I_{g}$, which is easily verified to be $\operatorname{Mod}_{g, 1}$-equivariant. The theorem follows.

\section{Skeleton of the proof of Lemma 3.3}

This section sets the stage for the remainder of the paper by reducing the proof of Lemma 3.3 to two further lemmas which are proven in the remaining sections. It also proves Lemma 3.4. This is all done in $\$ 5.2$. Before that, $\S 5.1$ sets up some notation which will be used in the remainder of the paper. 


\subsection{Notation for $K_{g}$}

In this section, we will introduce notation for elements of $\mathrm{H}_{1}\left(K_{g} ; \mathbb{Q}\right)$. Let $\rho: K_{g} \rightarrow \mathrm{H}_{1}\left(K_{g} ; \mathbb{Q}\right)$ be the abelianization map. Recall that the notation $g_{1}^{g_{2}}$ stands for $g_{2} g_{1} g_{2}^{-1}$. Observe that if $a \in K_{g}$ and $x, y \in \pi_{1}\left(\Sigma_{g}\right)$ are such that $x y^{-1} \in K_{g}$, then

$$
\rho\left(a^{x}\right)=\rho\left(a^{\left(x y^{-1}\right) y}\right)=\rho\left(\left(x y^{-1}\right)\left(a^{y}\right)\left(x y^{-1}\right)^{-1}\right)=\rho\left(x y^{-1}\right)+\rho\left(a^{y}\right)-\rho\left(x y^{-1}\right)=\rho\left(a^{y}\right) .
$$

This implies that for $a \in K_{g}$ and $x, y \in \pi_{1}\left(\Sigma_{g}\right)$ and $v \in H_{L}$, we may unambiguously define

$$
\langle\langle a\rangle\rangle^{\nu}=\rho\left(a^{\tilde{v}}\right) \quad \text { and } \quad\langle\langle x, y\rangle\rangle^{\nu}=\rho\left([x, y]^{\tilde{v}}\right),
$$

where $\tilde{v} \in \pi_{1}\left(\Sigma_{g}\right)$ is any lift of $v$ under the map $\pi_{1}\left(\Sigma_{g}\right) \rightarrow H_{L}$.

As another bit of notation, for $x \in \pi_{1}\left(\Sigma_{g}\right)$ denote by $\bar{x}$ the associated element of $H_{L}=\mathrm{H}_{1}\left(\Sigma_{g} ; \mathbb{Z} / L\right)$.

With this notation, we have the following identities.

\section{Lemma 5.1.}

1. For $a \in K_{g}$ and $x \in \pi_{1}\left(\Sigma_{g}\right)$, we have $\langle\langle a, x\rangle\rangle=\langle\langle a\rangle\rangle-\langle\langle a\rangle\rangle^{\bar{x}}$.

2. For $x, y \in \pi_{1}\left(\Sigma_{g}\right)$, we have $\langle\langle x, y\rangle\rangle=-\langle\langle y, x\rangle$.

3. For $x, y, z \in \pi_{1}\left(\Sigma_{g}\right)$, we have $\left.\langle\langle x y, z\rangle\rangle=\langle\langle x, z\rangle\rangle+\langle y y, z\rangle\right\rangle^{\bar{x}}$.

4. For $x, y \in \pi_{1}\left(\Sigma_{g}\right)$, we have $\left\langle\left\langle x^{-1}, y\right\rangle\right\rangle=-\langle\langle x, y\rangle\rangle^{-\bar{x}}$.

Proof. Items 1 and 2 are obvious, item 3 follows from the commutator identity $[x y, z]=[x, z][y, z]^{x}$, and item 4 follows from item 3 .

\subsection{Skeleton of the proof of Lemma 3.3}

Recall that we want to prove that the map

$$
\mathrm{H}_{1}\left(K_{g} ; C_{g, 1}\right) \rightarrow \mathrm{H}_{1}\left(\operatorname{Mod}_{g}^{1}(L) ; C_{g}\right)
$$

is the zero map. This map factors through $\mathrm{H}_{1}\left(K_{g} ; C_{g}\right)$. Since $K_{g}$ acts trivially on $C_{g}$, this latter group is isomorphic to $\mathrm{H}_{1}\left(K_{g} ; \mathbb{Z}\right) \otimes C_{g}$.

Our first order of business is to characterize the image of $\mathrm{H}_{1}\left(K_{g} ; C_{g, 1}\right)$ in $\mathrm{H}_{1}\left(K_{g} ; C_{g}\right)$. The long exact sequence in $K_{g}$-homology associated to the short exact sequence

$$
0 \longrightarrow I_{g} \longrightarrow C_{g, 1} \longrightarrow C_{g} \longrightarrow 0
$$

of $K_{g}$-modules contains the segment

$$
\mathrm{H}_{1}\left(K_{g} ; C_{g, 1}\right) \longrightarrow \mathrm{H}_{1}\left(K_{g} ; \mathbb{Z}\right) \otimes C_{g} \stackrel{\partial}{\longrightarrow} I_{g} .
$$

Here we have used the fact that $\mathrm{H}_{0}\left(K_{g} ; I_{g}\right)=\left(I_{g}\right)_{K_{g}}=I_{g}$. The image of $\mathrm{H}_{1}\left(K_{g} ; C_{g, 1}\right)$ in $\mathrm{H}_{1}\left(K_{g} ; C_{g}\right)$ is the kernel of $\partial$. It turns out that $\partial$ is closely related to the Reidemeister pairing $\omega(\cdot, \cdot)$ from Lemma 3.2.

Lemma 5.2. The map $\partial: \mathrm{H}_{1}\left(K_{g} ; \mathbb{Z}\right) \otimes C_{g} \rightarrow I_{g}$ is the restriction of the linear map $\mathrm{H}_{1}\left(K_{g} ; \mathbb{Q}\right) \otimes \mathrm{H}_{1}\left(K_{g} ; \mathbb{Q}\right) \rightarrow I_{g}$ induced by the bilinear Reidemeister pairing $\omega(\cdot, \cdot)$.

Proof. Consider $f \otimes y \in \mathrm{H}_{1}\left(K_{g} ; \mathbb{Z}\right) \otimes C_{g}$. Tracing through the construction of the long exact sequence (see, e.g., $[6, \S I I I .7])$, we can calculate $\partial(f \otimes y)$ as follows. Lift $y$ to $\tilde{y} \in C_{g, 1}$. Then

$$
\partial(f \otimes y)=f(\tilde{y})-\tilde{y} \in I_{g} .
$$

The lemma then follows from Lemma 3.2. 


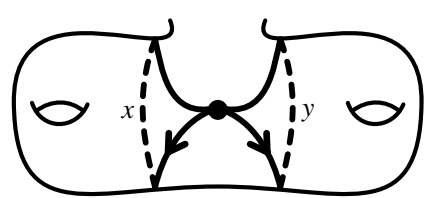

a

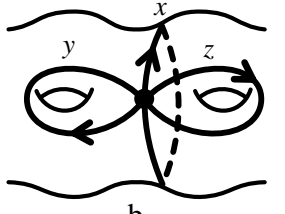

b

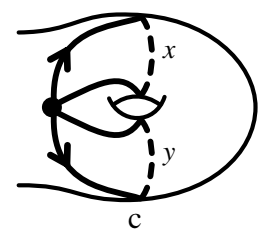

b. $\{x, y\}$ is strongly essentially separate from $\{x, z\} . \quad$ c. $x$

Figure 2: $a$. $x$ and $y$ are strongly essentially separate and $y$ are essentially separate but not strongly essentially separate

We thus want to determine which pairs of elements evaluate to zero under $\omega(\cdot, \cdot)$. This will require the following definitions.

Definition. Consider sets of curves $s=\left\{x_{1}, \ldots, x_{n}\right\} \subset \pi_{1}\left(\Sigma_{g}\right)$ and $s^{\prime}=\left\{y_{1}, \ldots, y_{m}\right\} \subset \pi_{1}\left(\Sigma_{g}\right)$.

- The sets $s$ and $s^{\prime}$ are essentially separate if there exist connected subsurfaces $X$ and $X^{\prime}$ of $\Sigma_{g}$ with the following properties.

- Both $X$ and $X^{\prime}$ contain the basepoint.

- $\Sigma_{g}=X \cup X^{\prime}$ and $X \cap X^{\prime}=\partial X=\partial X^{\prime}$.

- $s \subset \operatorname{Im}\left(\pi_{1}(X) \rightarrow \pi_{1}\left(\Sigma_{g}\right)\right)$ and $s^{\prime} \subset \operatorname{Im}\left(\pi_{1}\left(X^{\prime}\right) \rightarrow \pi_{1}\left(\Sigma_{g}\right)\right)$.

- The sets $s$ and $s^{\prime}$ are strongly essentially separate if we can choose $X$ and $X^{\prime}$ as above such that both $X$ and $X^{\prime}$ have exactly one boundary component (which necessarily contains the basepoint).

See Figure 2 for examples of curves that are essentially separate and strongly essentially separate.

This brings us to the following key definition. Recall that $H_{L}=\mathrm{H}_{1}\left(\Sigma_{g} ; \mathbb{Z} / L\right)$.

Definition. Define $\mathscr{S}_{g} \subset \mathrm{H}_{1}\left(K_{g} ; \mathbb{Q}\right) \times C_{g}$ to equal $\mathscr{S}_{g}(1) \cup \mathscr{S}_{g}(2)$, where the $\mathscr{S}_{g}(i)$ are as follows. To simplify our notation, we will denote $\pi_{1}\left(\Sigma_{g}\right)$ by $\pi$.

$$
\begin{aligned}
& \mathscr{S}_{g}(1)=\left\{\left(\langle\langle x\rangle\rangle^{v},\langle\langle y\rangle\rangle^{v^{\prime}}\right) \mid v, v^{\prime} \in H_{L}, x \in K_{g}, y \in[\pi, \pi] \text {, and } x \text { and } y \text { are essentially separate }\right\} \text {, } \\
& \mathscr{S}_{g}(2)=\left\{(\langle x\rangle\rangle^{v},\left\langle\left\langle y, z^{L}\right\rangle\right\rangle^{\nu^{\prime}}\right) \mid v, v^{\prime} \in H_{L}, x \in K_{g}, y, z \in \pi, z \text { can be realized by a simple } \\
& \text { closed nonseparating curve, and }\{z\} \text { and }\{x, y\} \text { are } \\
& \text { strongly essentially separate }\} \text {. }
\end{aligned}
$$

We now prove the following.

Lemma 5.3. For $(x, y) \in \mathscr{S}_{g}$, we have $\omega(x, y)=0$.

Proof. We must deal with both $\mathscr{S}_{g}(1)$ and $\mathscr{S}_{g}(2)$.

Step 1. $\omega\left(\langle\langle x\rangle\rangle^{v},\langle\langle y\rangle\rangle^{\prime}\right)=0$ for $\left(\langle\langle x\rangle\rangle^{v},\langle\langle y\rangle\rangle^{v^{\prime}}\right) \in \mathscr{S}_{g}(1)$.

Since $x$ and $y$ are essentially separate, they can be freely homotoped to disjoint curves. This implies that any two lifts of $x$ and $y$ to the cover of $\Sigma_{g}$ corresponding to $K_{g}$ can be homotoped so as to be disjoint. Examining the formula for $\omega(\cdot, \cdot)$ in Lemma 3.2, this immediately implies that $\omega\left(\langle\langle x\rangle\rangle^{v},\langle\langle y\rangle\rangle^{\prime}\right)=0$, as desired.

Step 2. $\omega\left(\langle\langle x\rangle\rangle^{v},\left\langle\left\langle y, z^{L}\right\rangle\right\rangle^{\prime}\right)=0$ for $\left(\langle\langle x\rangle\rangle^{v},\left\langle\left\langle y, z^{L}\right\rangle\right\rangle^{v^{\prime}}\right) \in \mathscr{S}_{g}(2)$.

Recall that if $w \in \pi_{1}\left(\Sigma_{g}\right)$, then $\bar{w}$ denotes the element of $H_{L}=\mathrm{H}_{1}\left(\Sigma_{g} ; \mathbb{Z} / L\right)$ associated to $w$. By Lemma 5.1 , we have

$$
\left\langle\left\langle y, z^{L}\right\rangle\right\rangle^{v^{\prime}}=\left\langle\left\langle z^{L}\right\rangle\right\rangle^{v^{\prime}+\bar{y}}-\left\langle\left\langle z^{L}\right\rangle\right\rangle^{\nu^{\prime}}
$$

Since $x$ and $z^{L}$ are essentially separate, an argument similar to the argument in Step 1 shows that

$$
\omega\left(\langle\langle x\rangle\rangle^{v},\left\langle\left\langle y, z^{L}\right\rangle\right\rangle^{v^{\prime}}\right)=\omega\left(\langle\langle x\rangle\rangle^{v},\left\langle\left\langle z^{L}\right\rangle\right\rangle^{v^{\prime}+\bar{y}}\right)-\omega\left(\langle\langle x\rangle\rangle^{v},\left\langle\left\langle z^{L}\right\rangle\right\rangle^{v^{\prime}}\right)=0-0=0,
$$

as desired. 
Remark. In Step 2 of the above, we only used the fact that $z$ is essentially separate from $x$. The remainder of the assumptions on elements of $\mathscr{S}_{g}(2)$ will be later used in the proof of Lemma 5.5.

It follows that the set $\left\{x \otimes y \mid(x, y) \in \mathscr{S}_{g}\right\}$ is contained in $\operatorname{ker}(\partial)$. This is not everything, however. Since $\operatorname{Mod}_{g}^{1}(L)$ acts trivially on $I_{g}$, the kernel of $\partial$ also contains $x \otimes y-f(x) \otimes f(y)$ for $x \in \mathrm{H}_{1}\left(K_{g} ; \mathbb{Z}\right)$ and $y \in C_{g}$ and $f \in \operatorname{Mod}_{g}^{1}(L)$. Define $\mathscr{K}_{g}<\mathrm{H}_{1}\left(K_{g} ; \mathbb{Z}\right) \otimes C_{g}$ be the span of the set

$$
\left\{x \otimes y \mid(x, y) \in \mathscr{S}_{g}\right\} \cup\left\{x \otimes y-f(x) \otimes f(y) \mid x \in \mathrm{H}_{1}\left(K_{g} ; \mathbb{Z}\right), y \in C_{g}, f \in \operatorname{Mod}_{g}^{1}(L)\right\}
$$

and let $Q_{g}=\mathrm{H}_{1}\left(K_{g} ; \mathbb{Z}\right) \otimes C_{g} / \mathscr{K}_{g}$. Since $\mathscr{K}_{g} \subset \operatorname{ker}(\partial)$, the map $\partial: \mathrm{H}_{1}\left(K_{g} ; \mathbb{Z}\right) \otimes C_{g} \rightarrow I_{g}$ induces a map $\psi: Q_{g} \rightarrow I_{g}$. Following preliminary results in $\S 6$, we will prove the following lemma in $\S 7$.

Lemma 5.4. For $g \geq 3$, the map $\psi$ is an isomorphism.

It follows that

$$
\mathscr{K}_{g}=\operatorname{ker}(\partial)=\operatorname{Im}\left(\mathrm{H}_{1}\left(K_{g} ; C_{g, 1}\right) \rightarrow \mathrm{H}_{1}\left(K_{g} ; C_{g}\right)\right) .
$$

To prove Lemma 3.3, which asserts that the map $\mathrm{H}_{1}\left(K_{g} ; C_{g, 1}\right) \rightarrow \mathrm{H}_{1}\left(\operatorname{Mod}_{g}^{1}(L) ; C_{g}\right)$ is the zero map, it therefore suffices to prove the following lemma, whose proof is in $\S 8$.

Lemma 5.5. For $g \geq 4$, the image of $\mathscr{K}_{g}$ in $\mathrm{H}_{1}\left(\operatorname{Mod}_{g}^{1}(L)\right.$; $\left.C_{g}\right)$ is zero.

This completes the outline of the proof of Lemma 3.3 (and our outline of the remainder of the paper). However, we also owe the reader a proof of Lemma 3.4, which asserts that

$$
\left(C_{g}\right)_{\pi_{1}\left(\Sigma_{g}\right)}=\left(C_{g, 1}\right)_{\pi_{1}\left(\Sigma_{g}\right)}=0
$$

for $g \geq 3$.

Proof of Lemma 3.4. We can extend the long exact sequence (8) to the right to get an exact sequence

$$
\mathrm{H}_{1}\left(K_{g} ; \mathbb{Z}\right) \otimes C_{g} \stackrel{\partial}{\longrightarrow} I_{g} \longrightarrow\left(C_{g, 1}\right)_{K_{g}} \longrightarrow C_{g} \longrightarrow 0 .
$$

Lemma 5.4 implies that $\partial$ is surjective, so we deduce that $\left(C_{g, 1}\right)_{K_{g}} \cong C_{g}$. It is thus enough to prove that $\left(C_{g}\right)_{\pi_{1}\left(\Sigma_{g}\right)}=0$. Lemma 2.2 implies that there is a $\pi_{1}\left(\Sigma_{g}\right)$-invariant decomposition

$$
\mathrm{H}_{1}\left(K_{g} ; \mathbb{Q}\right) \cong C_{g} \oplus \mathrm{H}_{1}\left(\Sigma_{g} ; \mathbb{Q}\right)
$$

Also, Lemma 2.3 implies that $\left(\mathrm{H}_{1}\left(K_{g} ; \mathbb{Q}\right)\right)_{\pi_{1}\left(\Sigma_{g}\right)} \cong \mathrm{H}_{1}\left(\Sigma_{g} ; \mathbb{Q}\right)$. We conclude that $\left(C_{g}\right)_{\pi_{1}\left(\Sigma_{g}\right)}=0$.

\section{Generators and relations for $Q_{g}$}

This section contains preliminaries for the proof of Lemma 5.4. We begin in $\S 6.1$ by introducing the notion of the intersection pattern of curves, which will play an important role in both this section and in $\S 8$. Next, in $\S 6.2$, we introduce certain important elements $X\left(v, w_{1}, w_{2}\right)$ of $Q_{g}$. We calculate the image of $X\left(v, w_{1}, w_{2}\right)$ under $\psi$ in $\S 6.3$. We show that the $X\left(v, w_{1}, w_{2}\right)$ span $Q_{g}$ in $\S 6.4$. Finally, in $\S 6.5$, we determine some relations between these elements.

Lemma 5.4 is proven in $\S 7$ below. The proof is essentially a lengthy calculation with generators and relations.

Throughout this section, let $\eta: \mathrm{H}_{1}\left(K_{g} ; \mathbb{Z}\right) \otimes C_{g} \rightarrow Q_{g}$ be the projection. 

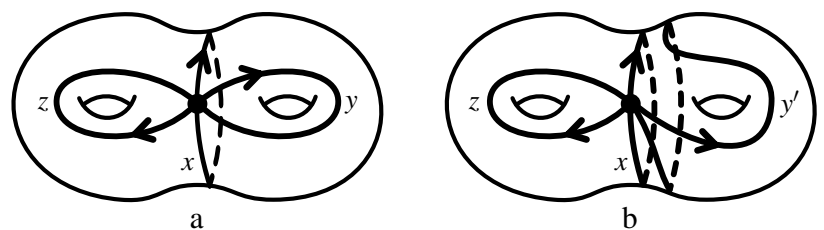

Figure 3: The curves $\{x, y, z\}$ in a have the same oriented intersection pattern as the curves $\left\{x, y^{\prime}, z\right\}$ in $b$.

\subsection{Intersection patterns}

We will have to perform some detailed calculations with elements of $\pi_{1}\left(\Sigma_{g}\right)$. These calculations will depend on certain pictures of curves on the surface, and in this section we will establish some vocabulary for this. We begin with the following definition.

Definition. Let $S$ be a surface, possibly with boundary. Assume that $S$ has a fixed basepoint. An embedding $i: S \rightarrow \Sigma_{g}$ is a simple embedding if $i$ takes the basepoint of $S$ to the basepoint of $\Sigma_{g}$ and all components of $\Sigma_{g} \backslash i(S)$ have one boundary component.

Remark. We allow $S=\Sigma_{g}$ and $i=$ id.

Remark. The key property of simple embeddings is as follows. Let $i: S \rightarrow \Sigma_{g}$ be a simple embedding. Then if $\gamma$ is a simple closed separating curve on $S$, then $i(\gamma)$ is a simple closed separating curve on $\Sigma_{g}$.

Next, we make the following definition.

Definition. Let $S$ be a surface, possibly with boundary, and let $\left\{x_{1}^{\prime}, \ldots, x_{k}^{\prime}\right\} \subset \pi_{1}(S)$. We will say that a set $\left\{x_{1}, \ldots, x_{k}\right\} \subset \pi_{1}\left(\Sigma_{g}\right)$ of curves has the same unoriented intersection pattern as $\left\{x_{1}^{\prime}, \ldots, x_{k}^{\prime}\right\}$ if there is a simple embedding $f: S \rightarrow \Sigma_{g}$ such that $f_{*}\left(x_{i}^{\prime}\right)=x_{i}^{ \pm 1}$ for all $1 \leq i \leq k$. If $f$ can be chosen such that $f\left(x_{i}^{\prime}\right)=x_{i}$ for all $1 \leq i \leq k$, then we will say that that the curves have the same oriented intersection pattern.

Remark. In what follows, the surface $S$ and the curves $\left\{x_{1}^{\prime}, \ldots, x_{k}^{\prime}\right\}$ will often be given by pictures. To avoid cluttering the pictures, we will often depict boundary components via gaps in their edges. For instance, there are boundary components at the top and bottom of Figure 4.a below.

We will frequently assert without proof that a set of curves has a given (un)oriented intersection pattern. In all these cases, the assertion will be a trivial consequence of the "change of coordinates" principle from $[8, \S 1.3]$. Rather than give a formal description of this principle, we will illustrate it with a concrete example (there are many more examples in $[8, \S 1.3]$ ). Namely, we will prove that the curves $\{x, y, z\}$ in Figure 3.a have the same oriented intersection pattern as the curves $\left\{x, y^{\prime}, z\right\}$ in Figure 3.b (we remark that $y^{\prime}=y^{-1} x$ ).

The proof is as follows. The union of the curves in Figure 3.a (resp. Figure 3.b) forms an oriented graph $\Gamma_{1}$ (resp. $\Gamma_{2}$ ) embedded in $\Sigma_{2}$ with one vertex (the basepoint) and three loops labeled with $\{x, y, z\}$ (resp. $\left\{x, y^{\prime}, z\right\}$ ). There is an isomorphism $f: \Gamma_{1} \rightarrow \Gamma_{2}$ taking the edge labeled $x$ to the edge labeled $x$, the edge labeled $y$ to the edge labeled $y^{\prime}$, and the edge labeled $z$ to the edge labeled $z$. The embedding of $\Gamma_{i}$ in $\Sigma_{2}$ induces a cyclic order on the oriented edges entering and leaving the single vertex, and the isomorphism $f$ respects these cyclic orderings. This implies that $f$ extends to a diffeomorphism $f^{\prime}: N_{1} \rightarrow N_{2}$, where $N_{i}$ is a regular neighborhood of $\Gamma_{i}$. An Euler characteristic computation shows that the components of $\Sigma_{2} \backslash N_{i}$ are diffeomorphic, and we thus obtain a basepoint-preserving diffeomorphism $f^{\prime \prime}: \Sigma_{2} \rightarrow \Sigma_{2}$ such that $\left(f^{\prime \prime}\right)_{*}(x)=x$ and $\left(f^{\prime \prime}\right)_{*}(y)=y^{\prime}$ and $\left(f^{\prime \prime}\right)_{*}(z)=z$, as desired.

\subsection{The elements $X\left(v, w_{1}, w_{2}\right)$}

The purpose of this section is to introduce certain elements $X\left(v, w_{1}, w_{2}\right)$ in $Q_{g}$. The key will be the following lemma. In it, recall that if $x \in \pi_{1}\left(\Sigma_{g}\right)$, then $\bar{x}$ denotes the element of $H_{L}=\mathrm{H}_{1}\left(\Sigma_{g} ; \mathbb{Z} / L\right)$ associated to $x$.

Lemma 6.1. Fix $w \in H_{L}=\mathrm{H}_{1}\left(\Sigma_{g} ; \mathbb{Z} / L\right)$. For $1 \leq i \leq 2$, let $x_{i}, y_{i}, z_{i} \in \pi_{1}\left(\Sigma_{g}\right)$ be such that $\left\{x_{i}, y_{i}, z_{i}\right\}$ has the same oriented intersection pattern as the curves $\{x, y, z\}$ in Figure 4.a. Assume that $\bar{y}_{1}=\bar{y}_{2}$ and $\bar{z}_{1}=\bar{z}_{2}$. Then $\eta\left(\left\langle\left\langle x_{1}\right\rangle\right\rangle \otimes\left\langle\left\langle y_{1}, z_{1}\right\rangle\right\rangle^{w}\right)=\eta\left(\left\langle\left\langle x_{2}\right\rangle\right\rangle \otimes\left\langle\left\langle y_{2}, z_{2}\right\rangle\right\rangle^{w}\right)$. 


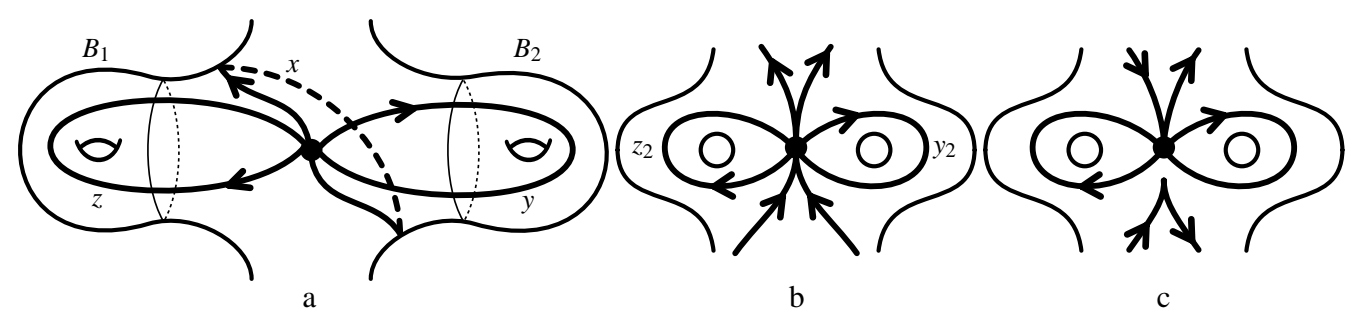

Figure 4: $a$. The configuration of curves such that $\left.\phi(\langle x\rangle\rangle\langle\langle y, z\rangle\rangle^{v}\right)=X\left(v, w_{1}, w_{2}\right)$. Also, the one-holed tori $B_{1}$ and $B_{2}$ will be needed in the proof of Lemma 6.3. The central four-holed sphere in the picture will be called A in that proof. $\quad b$. The curves $f\left(x_{1}\right)$ and $x_{2}$ leave at the top and come back at the bottom. $\quad c$. The product $f\left(x_{1}\right) x_{2}^{-1}$ is essentially disjoint from $\left[y_{2}, z_{2}\right]$. The orientations of the "top" and "bottom" piece depend on the manner in which $f\left(x_{1}\right)$ and $x_{2}$ leave and come back to the basepoint

Proof. It is easy to see that there exists some $f \in \operatorname{Mod}_{g}^{1}(L)$ such that $f\left(y_{1}\right)=y_{2}$ and $f\left(z_{1}\right)=z_{2}$ (the proof of this is a slight variation on the proof of [20, Proposition 6.7], which proves the analogous result for unbased curves). We then have

$$
\eta\left(\left\langle\left\langle x_{1}\right\rangle\right\rangle \otimes\left\langle\left\langle y_{1}, z_{1}\right\rangle\right\rangle^{w}\right)=\eta\left(\left\langle\left\langle f\left(x_{1}\right)\right\rangle\right\rangle \otimes\left\langle\left\langle f\left(y_{1}\right), f\left(z_{1}\right)\right\rangle\right\rangle^{w}\right)=\eta\left(\left\langle\left\langle f\left(x_{1}\right)\right\rangle \otimes \otimes\left\langle y_{2}, z_{2}\right\rangle\right\rangle^{w}\right) .
$$

The curves $\left\{f\left(x_{1}\right), y_{2}, z_{2}\right\}$ have the same oriented intersection pattern as the curves $\{x, y, z\}$ in Figure 4.a. Moreover (see Figures 4.b-c), the curves $f\left(x_{1}\right) x_{2}^{-1}$ and $\left[y_{2}, z_{2}\right]$ are essentially separate, so we conclude that

$$
\eta\left(\left\langle\left\langle f\left(x_{1}\right)\right\rangle\right\rangle \otimes\left\langle\left\langle y_{2}, z_{2}\right\rangle\right\rangle^{w}\right)=\eta\left(\left\langle\left\langle x_{2}\right\rangle\right\rangle \otimes\left\langle\left\langle y_{2}, z_{2}\right\rangle\right\rangle^{w}\right),
$$

as desired.

We will need the following definition. Let $i(\cdot, \cdot)$ be the $\mathbb{Z} / L$-valued algebraic intersection pairing on $H_{L}$.

Definition. A $k$-element set $\left\{w_{1}, \ldots, w_{k}\right\} \subset H_{L}$ will be said to be isotropic if $i\left(w_{i}, w_{j}\right)=0$ for all $1 \leq i, j \leq k$ and unimodular if $\left\langle w_{1}, \ldots, w_{k}\right\rangle$ is direct summand of $H_{L}$ that is isomorphic to a $k$-dimensional free $\mathbb{Z} / L$ submodule.

It is clear that if the curves $\{x, y, z\}$ have the same oriented intersection pattern as the curves in Figure 4.a, then $\{\bar{y}, \bar{z}\} \subset H_{L}$ is isotropic and unimodular. The converse is true as well. This will require the following lemma.

Lemma 6.2. For some $n, m \geq 0$, let $\left\{w_{1}, \ldots, w_{n}, w_{1}^{\prime}, \ldots, w_{m}^{\prime}\right\} \subset H_{L}$ be a unimodular set. Assume that

$$
i\left(w_{i}, w_{j}\right)=i\left(w_{i^{\prime}}^{\prime}, w_{j^{\prime}}^{\prime}\right)=0 \quad \text { and } \quad i\left(w_{i}, w_{i^{\prime}}\right)= \begin{cases}1 & \text { if } i=i^{\prime} \\ 0 & \text { if } i \neq i^{\prime}\end{cases}
$$

for $1 \leq i, j \leq n$ and $1 \leq i^{\prime}, j^{\prime} \leq m$. There then exists a set $\left\{\alpha_{1}, \ldots, \alpha_{n}, \alpha_{1}^{\prime}, \ldots, \alpha_{m}^{\prime}\right\}$ of unbased oriented simple closed curves on $\Sigma_{g}$ with the following properties.

- The $\mathbb{Z} / L$-homology class of $\alpha_{i}$ is $w_{i}$ for $1 \leq i \leq n$ and the $\mathbb{Z} / L$-homology class of $\alpha_{i}^{\prime}$ is $w_{i}^{\prime}$ for $1 \leq i \leq m$.

- $\alpha_{i}$ and $\alpha_{i}^{\prime}$ intersect once for $1 \leq i \leq \min (n, m)$. Otherwise, the curves $\left\{\alpha_{1}, \ldots, \alpha_{n}, \alpha_{1}^{\prime}, \ldots, \alpha_{m}^{\prime}\right\}$ are pairwise disjoint.

Proof. Identical to the proof of [19, Lemma A.3].

If $\left\{w_{1}, w_{2}\right\} \subset H_{L}$ is isotropic and unimodular, then Lemma 6.2 says that we can find unbased, disjoint simple closed curves $Y$ and $Z$ such that the $\mathbb{Z} / L$-homology classes of $Y$ and $Z$ are $w_{1}$ and $w_{2}$, respectively. Connecting $Y$ and $Z$ to the basepoint in an appropriate way, we find $y, z \in \pi_{1}\left(\Sigma_{g}\right)$ such that $\bar{y}=w_{1}$ and $\bar{z}=w_{2}$ and $\{y, z\}$ has the same oriented intersection pattern as the curves in Figure 4.a. It is then clear that we can find some $x \in \pi_{1}\left(\Sigma_{g}\right)$ such that $\{x, y, z\}$ has the same oriented intersection pattern as the curves in Figure 4.a.

We now introduce notation for the elements of $Q_{g}$ we have been discussing. 
Definition. For $v, w_{1}, w_{2} \in H_{L}$ such that $\left\{w_{1}, w_{2}\right\}$ is isotropic and unimodular, define $X\left(v, w_{1}, w_{2}\right)=\eta(\langle x\rangle\rangle \otimes$ $\left.\langle\langle y, z\rangle\rangle^{\nu}\right) \in Q_{g}$, where $x, y, z \in \pi_{1}\left(\Sigma_{g}\right)$ have the same oriented intersection pattern as the curves in Figure 4.a and $w_{1}=\bar{y}$ and $w_{2}=\bar{z}$.

The paragraph before the definition shows that we can find appropriate $\{x, y, z\}$, and Lemma 6.1 implies that $X\left(v, w_{1}, w_{2}\right)$ only depends on $\left\{v, w_{1}, w_{2}\right\}$.

\subsection{The image of $X\left(v, w_{1}, w_{2}\right)$ under $\psi$}

Recall that $\psi$ is the natural map $Q_{g} \rightarrow I_{g}$ induced by the Reidemeister pairing $\omega(\cdot, \cdot)$ from Lemma 3.2. We now prove the following.

Lemma 6.3. Consider $v, w_{1}, w_{2} \in H_{L}$ such that $\left\{w_{1}, w_{2}\right\}$ is isotropic and unimodular. Then $\psi\left(X\left(v, w_{1}, w_{2}\right)\right)=$ $\llbracket v \rrbracket-\llbracket v+w_{1} \rrbracket-\llbracket v+w_{2} \rrbracket+\llbracket v+w_{1}+w_{2} \rrbracket$.

Proof. Let $x, y, z \in \pi_{1}\left(\Sigma_{g}\right)$ be curves with the same oriented intersection pattern as the curves in Figure 4.a such that $\bar{y}=w_{1}$ and $\bar{z}=w_{2}$. We then have $\left.\left.X\left(v, w_{1}, w_{2}\right)=\eta(\langle x\rangle\rangle \otimes\langle y, z\rangle\right\rangle^{v}\right)$, and the lemma is equivalent to proving that

$$
\omega\left(\left\langle\langle x\rangle,\langle\langle y, z\rangle\rangle^{v}\right)=\llbracket v \rrbracket-\llbracket v+\bar{y} \rrbracket-\llbracket v+\bar{z} \rrbracket+\llbracket v+\bar{y}+\bar{z} \rrbracket .\right.
$$

Let $\rho: S_{g}^{K} \rightarrow \Sigma_{g}$ be the cover corresponding to $K_{g}$. The group of deck transformations is thus $H_{L}$. Let $B_{1} \subset \Sigma_{g}$ (resp. $\left.B_{2} \subset \Sigma_{g}\right)$ ) be the one-holed torus on the left (resp. right) side of Figure 4.a. Also, let $A \subset \Sigma_{g}$ be the four-holed sphere "between" $B_{1}$ and $B_{2}$ in Figure 4.a. We then have the following.

- $\rho^{-1}(A)$ is the disjoint union of $\left|H_{L}\right|=L^{2 g}$ four-holed spheres each of which projects homeomorphically onto $A$.

- $\rho^{-1}\left(B_{i}\right)$ is the disjoint union of $\left|H_{L}\right| / L^{2}=L^{2 g-2}$ components. If $\tilde{B}_{i}$ is one of those components, then $\tilde{B}_{i}$ is an $L^{2}$-holed torus and $\left.\rho\right|_{\tilde{B}_{i}}: \tilde{B}_{i} \rightarrow B_{i}$ is a cover with deck group $(\mathbb{Z} / L)^{2}$.

The homology class $\langle\langle y, z\rangle\rangle^{\nu}$ on $S_{g}^{K}$ can be realized by a simple closed curve $\gamma$ as in Figure 5 . If $\tilde{A}$ is the component of $\rho^{-1}(A)$ containing the basepoint, then this simple closed curve does the following.

- Beginning in the component $\llbracket v \rrbracket \cdot \tilde{A}$ of $\rho^{-1}(A)$, it goes through a component of $\rho^{-1}\left(B_{2}\right)$ to arrive in $\llbracket v+\bar{y} \rrbracket \cdot \tilde{A}$.

- It then goes through a component of $\rho^{-1}\left(B_{1}\right)$ to arrive in $\llbracket v+\bar{y}+\bar{z} \rrbracket \cdot \tilde{A}$.

- It then goes through a component of $\rho^{-1}\left(B_{2}\right)$ to arrive in $\llbracket v+\bar{z} \rrbracket \cdot \tilde{A}$.

- It finally goes through a component of $\rho^{-1}\left(B_{1}\right)$ to arrive back in $\llbracket v \rrbracket \cdot \tilde{A}$.

Let $\tilde{x}$ be the lift of $x$ contained in $\tilde{A}$. As is evident from Figure 5, the curve $\gamma$ intersects four different $H_{L^{-}}$ translates of $\tilde{x}$, two with positive sign and two with negative sign. Examining these intersections, we see that

$$
\omega\left(\left\langle\langle x\rangle,\langle\langle y, z\rangle\rangle^{v}\right)=\llbracket v \rrbracket-\llbracket v+\bar{y} \rrbracket-\llbracket v+\bar{z} \rrbracket+\llbracket v+\bar{y}+\bar{z} \rrbracket,\right.
$$

as desired. 

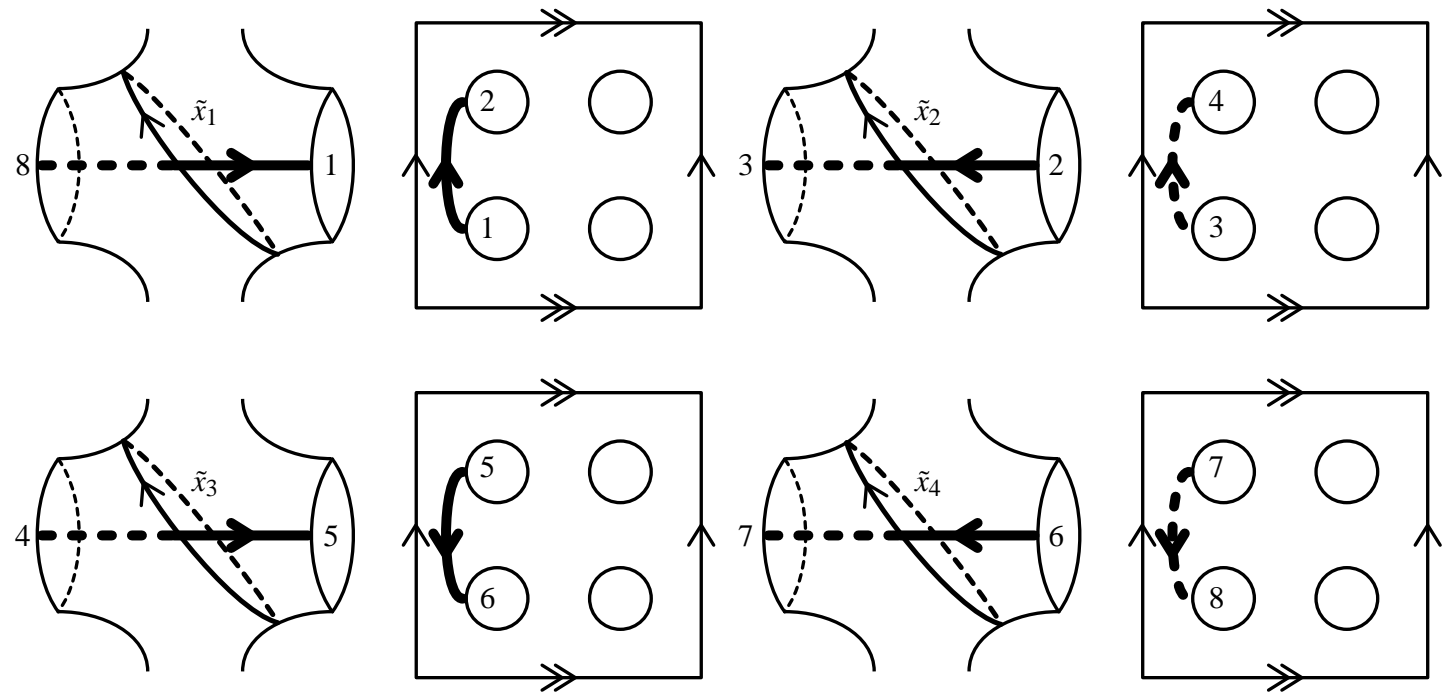

Figure 5: The curve $\gamma$ in $S_{g}^{K}$ whose homology class is $\langle\langle y, z\rangle\rangle^{\nu}$. The dark portions are the lifts of $y^{ \pm 1}$ and the dashed portions are the lifts of $z^{ \pm 1}$. The numbers at the end of each segment indicate where the curve goes next. The 4-holed spheres are components of $\rho^{-1}(A)$ and the $L^{2}$-holed tori (depicted here for $L=2$; the sides of the squares should be glued up in the indicated ways) are components of $\rho^{-1}\left(B_{1}\right)$ and $\rho^{-1}\left(B_{2}\right)$. The curve $\gamma$ intersects four lifts $\tilde{x}_{1}, \ldots, \tilde{x}_{4}$ of $\rho^{-1}(x)$. If $\tilde{x}$ is the lift of $x$ starting at the basepoint, then $\tilde{x}_{1}=\llbracket v \rrbracket \cdot \tilde{x}$ and $\tilde{x}_{2}=\llbracket v+\bar{y} \rrbracket \cdot \tilde{x}$ and $\tilde{x}_{3}=\llbracket v+\bar{y}+\bar{z} \rrbracket \cdot \tilde{x}$ and $\tilde{x}_{4}=\llbracket v+\bar{z} \rrbracket \cdot \tilde{x}$

\section{4 $Q_{g}$ is spanned by the $X\left(v, w_{1}, w_{2}\right)$}

In this section, we prove that the $X\left(v, w_{1}, w_{2}\right)$ span $Q_{g}$. The proof will use the following lemma.

Lemma 6.4 ([19, Lemma A.1]). Fix $g \geq 1$ and set $\pi=\pi_{1}\left(\Sigma_{g}\right)$. The group $[\pi, \pi]$ is then generated by the set $\{\gamma \mid \gamma \in \pi$ can be realized by a simple closed separating curve $\}$.

We now prove our lemma.

Lemma 6.5. For $g \geq 1$, the vector space $Q_{g}$ is spanned by the set

$$
\left\{X\left(v, w_{1}, w_{2}\right) \mid v, w_{1}, w_{2} \in H_{L},\left\{w_{1}, w_{2}\right\} \text { is isotropic and unimodular }\right\} .
$$

Proof. Define

$$
C_{g}(\mathbb{Z})=\operatorname{ker}\left(\mathrm{H}_{1}\left(K_{g} ; \mathbb{Z}\right) \longrightarrow \mathrm{H}_{1}\left(\Sigma_{g} ; \mathbb{Z}\right)\right) .
$$

Our proof will have three steps. As notation, for $q, q^{\prime} \in Q_{g}$, write $q \equiv q^{\prime}$ if $q$ and $q^{\prime}$ are equal modulo $\eta\left(C_{g}(\mathbb{Z}) \otimes C_{g}\right)$.

Step 1. Let $x, y, z \in \pi_{1}\left(\Sigma_{g}\right)$ be such that $z$ can be realized by a simple closed nonseparating curve and $\{z\}$ is strongly essentially separate from $\{x, y\}$. Also, let $v \in H_{L}$. Then $\eta\left(\left\langle\left\langle x^{L}\right\rangle\right\rangle \otimes\langle\langle y, z\rangle\rangle^{v}\right) \equiv 0$.

Since $\operatorname{Mod}_{g}^{1}(L)$ contains all inner automorphisms of $\pi_{1}\left(\Sigma_{g}\right)$, we can use the $\operatorname{Mod}_{g}^{1}(L)$-invariance of $Q_{g}$ to deduce that

$$
\eta\left(\left\langle\left\langle x^{L}\right\rangle\right\rangle \otimes\langle\langle y, z\rangle\rangle^{v+j \cdot \bar{z}}\right)=\eta\left(\left\langle\left\langle z^{-j} x^{L} z^{j}\right\rangle\right\rangle \otimes\langle\langle y, z\rangle\rangle^{v}\right) \equiv \eta\left(\left\langle\left\langle x^{L}\right\rangle\right\rangle \otimes\langle\langle y, z\rangle\rangle^{v}\right) .
$$

for all $j \in \mathbb{Z}$. Consequently,

$$
\eta\left(\left\langle\left\langle x^{L}\right\rangle\right\rangle \otimes\langle\langle y, z\rangle\rangle^{v}\right) \equiv \frac{1}{L} \sum_{j=0}^{L-1} \eta\left(\langle\langle x\rangle\rangle^{L} \otimes\langle\langle y, z\rangle\rangle^{v+j \cdot \bar{z}}\right)=\frac{1}{L} \eta\left(\left\langle\left\langle x^{L}\right\rangle\right\rangle \otimes\left\langle\left\langle y, z^{L}\right\rangle\right\rangle^{v}\right)=0 .
$$

This last equality follows from the fact that $\left(\left\langle\left\langle x^{L}\right\rangle\right\rangle,\left\langle\left\langle y, z^{L}\right\rangle\right\rangle^{v}\right) \in \mathscr{S}_{g}(2)$ 


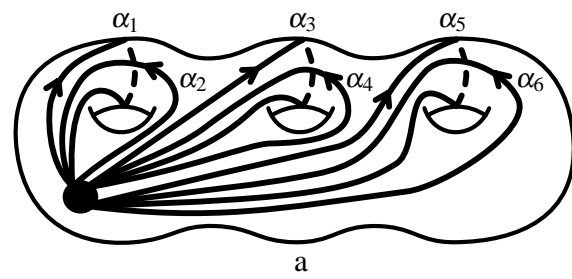

Figure 6: $a$. A standard basis for $\pi_{1}\left(\Sigma_{g}\right)$

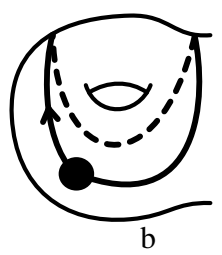

b. The curve $\left[\alpha_{1}, \alpha_{2}\right]$.

Step 2. $\eta\left(C_{g}(\mathbb{Z}) \otimes C_{g}\right)=Q_{g}$.

Our goal is to show that $q \equiv 0$ for all $q \in Q_{g}$. To do this, it is enough to show that $\eta\left(\left\langle\left\langle\alpha_{1}^{L}\right\rangle\right\rangle \otimes c\right) \equiv 0$ for all $\alpha_{1} \in \pi_{1}\left(\Sigma_{g}\right)$ that can be realized by a simple closed nonseparating curve and all $c \in C_{g}$. Extend $\alpha_{1}$ to a standard basis $\left\{\alpha_{1}, \ldots, \alpha_{2 g}\right\}$ for $\pi_{1}\left(\Sigma_{g}\right)$ as in Figure 6.a. The vector space $C_{g}$ is spanned by the set

$$
\left.S=\left\{\left\langle\alpha_{i}, \alpha_{j}\right\rangle\right\rangle^{v} \mid 1 \leq i<j \leq 2 g, v \in H_{L}\right\} .
$$

Consider $\left\langle\left\langle\alpha_{i}, \alpha_{j}\right\rangle\right\rangle^{v} \in S$. If $i \geq 3$ or $(i, j)=(1,2)$, then $\left[\alpha_{i}, \alpha_{j}\right]$ is essentially separate from $\alpha_{1}^{L}$ (see Figure 6.b for $(i, j)=(1,2))$ and thus $\eta\left(\left\langle\left\langle x^{L}\right\rangle\right\rangle\left\langle\left\langle\alpha_{i}, \alpha_{j}\right\rangle\right\rangle^{v}\right)=0$. Otherwise, $i \in\{1,2\}$ and $j>2$. It follows that $\left\{\alpha_{j}\right\}$ is strongly essentially separate from $\left\{\alpha_{1}, \alpha_{i}\right\}$, and thus Step 1 implies $\left.\eta\left(\left\langle\alpha_{1}^{L}\right\rangle\right\rangle \otimes\left\langle\left\langle\alpha_{i}, \alpha_{j}\right\rangle\right\rangle^{v}\right) \equiv 0$.

Step 3. $\eta\left(C_{g}(\mathbb{Z}) \otimes C_{g}\right)$ is generated by the set

$$
\left\{X\left(v, w_{1}, w_{2}\right) \mid v, w_{1}, w_{2} \in H_{L},\left\{w_{1}, w_{2}\right\} \text { is isotropic and unimodular }\right\} .
$$

Set

$$
S=\left\{\gamma \in \pi_{1}\left(\Sigma_{g}\right) \mid \gamma \neq 1, \gamma \text { can be realized by a simple closed separating curve }\right\} .
$$

Lemma 6.4 implies that $C_{g}(\mathbb{Z})$ is generated by $\{\langle\langle\gamma\rangle| \gamma \in S\}$. Consider $\gamma \in S$. Let $X_{1}$ and $X_{2}$ be the two surfaces into which $\gamma$ cuts $\Sigma_{g}$. Order them so that $X_{1}$ lies to the right of $\gamma$ and $X_{2}$ to the left. We can then find a basis $B_{\gamma}^{1} \cup B_{\gamma}^{2}$ for $\pi_{1}\left(\Sigma_{g}\right)$ with the following properties.

- For $\delta \in B_{\gamma}^{i}$, we have $\delta \in \operatorname{Im}\left(\pi_{1}\left(X_{i}\right) \rightarrow \pi_{1}\left(\Sigma_{g}\right)\right)$.

- Consider $\delta_{1} \in B_{\gamma}^{1}$ and $\delta_{2} \in B_{\gamma}^{2}$. The curves $\left\{\gamma, \delta_{1}, \delta_{2}\right\}$ then have the same oriented intersection pattern as the curves in Figure 4.a.

It follows that $C_{g}$ is spanned by the set $U_{\gamma} \cup V_{\gamma}$, where

$$
\left.\left.U_{\gamma}=\left\{\left\langle\delta_{1}, \delta_{2}\right\rangle\right\rangle^{v} \mid \delta_{i} \in B_{\gamma}^{i}, v \in H_{L}\right\} \quad \text { and } \quad V_{\gamma}=\left\{\left\langle\delta, \delta^{\prime}\right\rangle\right\rangle^{v} \mid \text { there exists } i \text { such that } \delta, \delta^{\prime} \in B_{\gamma}^{i}, v \in H_{L}\right\} \text {. }
$$

We then have that $C_{g}(\mathbb{Z}) \otimes C_{g}$ is spanned by the set $Z \cup W$, where

$$
\left.Z=\left\{\langle\langle\gamma\rangle\rangle \otimes c \mid \gamma \in S, c \in U_{\gamma}\right\} \quad \text { and } \quad W=\{\langle\gamma\rangle\rangle \otimes c \mid \gamma \in S, c \in V_{\gamma}\right\} .
$$

For $\left.\langle\langle\gamma\rangle\rangle \otimes\left\langle\delta_{1}, \delta_{2}\right\rangle\right\rangle^{v} \in Z$, we have $\eta\left(\left\langle\langle\gamma\rangle \otimes\left\langle\left\langle\delta_{1}, \delta_{2}\right\rangle\right\rangle^{\nu}\right)=X\left(v, \bar{\delta}_{1}, \bar{\delta}_{2}\right)\right.$. For $\langle\langle\gamma\rangle\rangle \otimes\left\langle\left\langle\delta, \delta^{\prime}\right\rangle\right\rangle^{v} \in W$, the curves $\gamma$ and $\left[\delta, \delta^{\prime}\right]$ are essentially separate, so $\left.\eta(\langle\gamma\rangle\rangle \otimes\left\langle\left\langle\delta, \delta^{\prime}\right\rangle\right\rangle^{v}\right)=0$. The desired result follows.

\subsection{Relations between the $X\left(w, v_{1}, v_{2}\right)$}

The goal of this section is to prove the following lemma, which gives relations between the $X\left(v, w_{1}, w_{2}\right)$.

Lemma 6.6. Let $\left\{w_{1}, w_{2}\right\} \subset \mathrm{H}_{1}\left(\Sigma_{g} ; \mathbb{Z} / L\right)$ be an isotropic and unimodular set. Then the following hold for all $v \in \mathrm{H}_{1}\left(\Sigma_{g} ; \mathbb{Z} / L\right)$.

$$
\text { 1. } X\left(v, w_{1}, w_{2}\right)=X\left(v, w_{2}, w_{1}\right)
$$



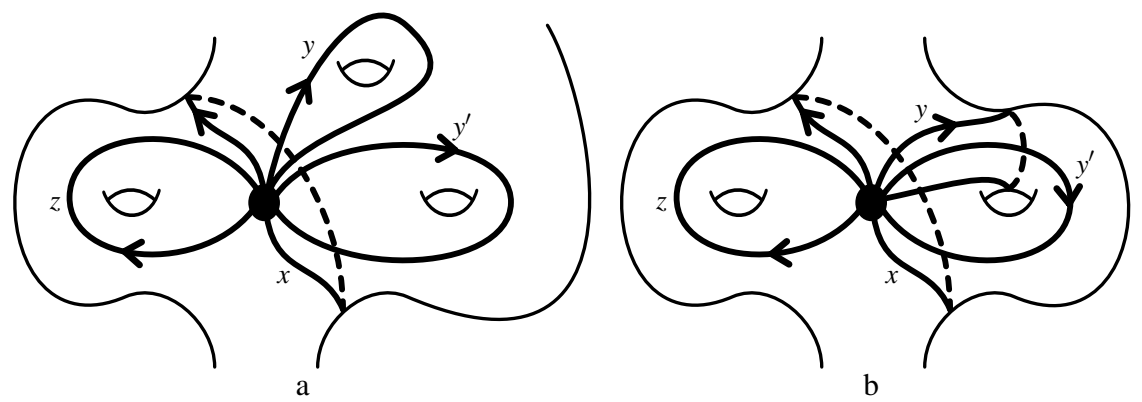

Figure 7: $a$. $i\left(y, y^{\prime}\right)=0$ and the sets of curves $\{x, y, z\}$ and $\left\{x, y^{\prime}, z\right\}$ and $\left\{x, y y^{\prime}, z\right\}$ have the same oriented intersection pattern as the curves in Figure 4.a $\left\{x, y\left(y^{\prime}\right)^{-1}, z\right\}$ have the same oriented intersection pattern as the curves in Figure 4.a

2. $X\left(v,-w_{1}, w_{2}\right)=-X\left(v-w_{1}, w_{1}, w_{2}\right)$

3. $\sum_{i=0}^{L-1} X\left(v+i \cdot w_{1}, w_{1}, w_{2}\right)=0$ and $\sum_{i=0}^{L-1} X\left(v+i \cdot w_{2}, w_{1}, w_{2}\right)=0$

4. Let $w_{3} \in \mathrm{H}_{1}\left(\Sigma_{g} ; \mathbb{Z} / L\right)$ be such that $\left\{w_{1}, w_{2}, w_{3}\right\}$ is unimodular, such that $i\left(w_{2}, w_{3}\right)=0$, and such that $-1 \leq i\left(w_{1}, w_{3}\right) \leq 1$. Then $X\left(v, w_{1}+w_{3}, w_{2}\right)=X\left(v, w_{1}, w_{2}\right)+X\left(v+w_{1}, w_{3}, w_{2}\right)$.

Remark. It is instructive to check (using the formula in Lemma 6.3) that each of these relations is taken to 0 by $\psi$.

Proof of Lemma 6.6. Let $x, y, z \in \pi_{1}\left(\Sigma_{g}\right)$ be curves such that $\{x, y, z\}$ has the same oriented intersection pattern as the curves in Figure 4.a and such that $\bar{y}=w_{1}$ and $\bar{z}=w_{2}$. Hence $X\left(v, w_{1}, w_{2}\right)=\eta\left(\left\langle\langle x\rangle \otimes\langle\langle y, z\rangle\rangle^{v}\right)\right.$.

For item 1, observe that if we flip $y$ and $z$, then our curves no longer have the same oriented intersection pattern as the curves in Figure 4.a (they do have the same unoriented intersection pattern). To restore the correct orientations, we must reverse $x$. In other words,

$$
X\left(v, w_{2}, w_{1}\right)=\eta\left(\left\langle\left\langle x^{-1}\right\rangle\right\rangle \otimes\langle\langle z, y\rangle\rangle^{v}\right)=\eta\left(\left(-\langle\langle x\rangle) \otimes\left(-\langle\langle y, z\rangle\rangle^{v}\right)\right)=\eta(\langle x\rangle\rangle \otimes\langle\langle y, z\rangle\rangle^{v}\right)=X\left(v, w_{1}, w_{2}\right),
$$

as desired.

For item 2, observe that the set of curves $\left\{x, y^{-1} x, z\right\}$ has the same oriented intersection pattern as $\{x, y, z\}$ (see the example in $\S 6.1$ ). Also, since $\bar{x}=0$, we have $\overline{y^{-1} x}=-\bar{y}=-w_{1}$. Thus we can apply Lemma 5.1 to get that

$$
\begin{aligned}
X\left(v,-w_{1}, w_{2}\right) & \left.=\eta\left(\langle\langle x\rangle\rangle\left\langle\left\langle y^{-1} x, z\right\rangle\right\rangle^{v}\right)=\eta(\langle x\rangle\rangle \otimes\left\langle\left\langle y^{-1}, z\right\rangle\right\rangle^{v}\right)+\eta\left(\left\langle\langle x\rangle \otimes\langle\langle x, z\rangle\rangle^{v-w_{1}}\right)\right. \\
& \left.=-\eta\left(\langle\langle x\rangle\rangle \otimes\langle\langle y, z\rangle\rangle^{v-w_{1}}\right)+\eta(\langle x\rangle\rangle \otimes\langle\langle x\rangle\rangle^{v-w_{1}}\right)-\eta\left(\langle\langle x\rangle\rangle \otimes\langle\langle x\rangle\rangle^{\nu-w_{1}+w_{2}}\right) .
\end{aligned}
$$

Since $x$ is essentially separate from itself, the last two terms vanish and this equals

$$
\left.-\eta(\langle x\rangle\rangle \otimes\langle\langle y, z\rangle\rangle^{\nu-w_{1}}\right)=-X\left(v-w_{1}, w_{1}, w_{2}\right)
$$

as desired.

For item 3, item 1 implies that it is enough to prove that $\sum_{i=0}^{L-1} X\left(v+i \cdot w_{2}, w_{1}, w_{2}\right)=0$. Observe that $\left(\langle\langle x\rangle\rangle,\left\langle\left\langle y, z^{L}\right\rangle\right\rangle^{v}\right) \in \mathscr{S}_{g}(2)$, so by Lemma 5.1 we have

$$
0=\eta\left(\langle\langle x\rangle\rangle \otimes\left\langle\left\langle y, z^{L}\right\rangle\right\rangle^{v}\right)=\sum_{i=0}^{L-1} \eta\left(\langle\langle x\rangle\rangle \otimes\langle\langle y, z\rangle\rangle^{v+i \cdot \bar{z}}\right)=\sum_{i=0}^{L-1} X\left(v+i \cdot w_{2}, w_{1}, w_{2}\right),
$$

as desired.

We conclude with item 4 . Here we will have to change our curves $x$ and $y$ and $z$. We will prove shortly that we can find $x, y, y^{\prime}, z \in \pi_{1}\left(\Sigma_{g}\right)$ with the following properties. 
- $\bar{y}=w_{1}$ and $\bar{y}^{\prime}=w_{3}$ and $\bar{z}=w_{2}$.

- The sets of curves $\{x, y, z\}$ and $\left\{x, y^{\prime}, z\right\}$ and $\left\{x, y y^{\prime}, z\right\}$ each have the same oriented intersection pattern as the curves in Figure 4.a.

See Figures 7.a,b. Assuming this for the moment, the proof is completed by appealing to Lemma 5.1 to deduce that

$$
\begin{aligned}
X\left(v, w_{1}+w_{3}, w_{2}\right) & \left.=\eta\left(\langle\langle x\rangle\rangle \otimes\left\langle\left\langle y y^{\prime}, z\right\rangle\right\rangle^{v}\right)=\eta\left(\langle\langle x\rangle\rangle \otimes\langle\langle y, z\rangle\rangle^{v}\right)+\eta(\langle x\rangle\rangle \otimes\left\langle\left\langle y^{\prime}, z\right\rangle\right\rangle^{v+\bar{y}}\right) \\
& =X\left(v, w_{1}, w_{2}\right)+X\left(v+w_{1}, w_{3}, w_{2}\right) .
\end{aligned}
$$

It remains to prove the above claim. By Lemma 6.2, we can find unbased oriented simple closed curves $Y$ and $Y^{\prime}$ and $Z$ on $\Sigma_{g}$ with the following properties.

- The $\mathbb{Z} / L$-homology classes of $Y, Y^{\prime}$, and $Z$ are $w_{1}, w_{3}$, and $w_{2}$, respectively.

- $Z$ is disjoint from $Y$ and $Y^{\prime}$. Also, $Y$ and $Y^{\prime}$ are disjoint if $i\left(w_{1}, w_{3}\right)=0$ and intersect once if $i\left(w_{1}, w_{3}\right)=$ \pm 1 .

We can then connect $Y, Y^{\prime}$, and $Z$ to the basepoint to obtain curves $y, y^{\prime}, z \in \pi_{1}\left(\Sigma_{g}\right)$ such that $\left\{y, y^{\prime}, z\right\}$ has the same oriented intersection pattern as the curves in Figure 7.a (if $i\left(w_{1}, w_{3}\right)=0$ ) or 7.b (if $i\left(w_{1}, w_{3}\right)=-1$ ) or 7.b with the orientation of the curve $y^{\prime}$ reversed (if $\left.i\left(w_{1}, w_{3}\right)=1\right)$. It is then clear that we can find $x \in \pi_{1}\left(\Sigma_{g}\right)$ such that $\left\{x, y, y^{\prime}, z\right\}$ has the indicated oriented intersection pattern, as desired.

\section{The map $\psi: Q_{g} \rightarrow I_{g}$ is an isomorphism}

The purpose of this section is to prove that the map $\psi: Q_{g} \rightarrow I_{g}$ is an isomorphism. The actual proof is in $\S 7.2$. This is proceeded by $\S 7.1$, which constructs a generating set $V$ for $Q_{g}$ that is slightly smaller than the generating set determined in $\S 6.4$.

Throughout this section, we will freely use the main results of $\S 6$ (i.e. Lemmas 6.3 and 6.5 and 6.6).

\subsection{A smaller generating set}

Fix a symplectic basis $B=\left\{a_{1}, b_{1}, \ldots, a_{g}, b_{g}\right\}$ for $H_{L}$. Define $V=V_{1} \cup V_{2} \subset Q_{g}$, where the $V_{i}$ are as follows.

$$
\begin{aligned}
& V_{1}=\left\{X\left(v, s_{1}, s_{2}\right) \mid v \in H_{L}, s_{1}, s_{2} \in B \text { distinct, } i\left(s_{1}, s_{2}\right)=0\right\}, \\
& \left.V_{2}=\left\{X\left(v, s_{1}, s_{1}+e s_{2}\right) \mid v \in H_{L}\right), s_{1}, s_{2} \in B \text { distinct, } e \in\{-1,1\}, i\left(s_{1}, s_{2}\right)=0\right\},
\end{aligned}
$$

The goal of this section is to prove the following.

Lemma 7.1. For $g \geq 4$, the vector space $Q_{g}$ is spanned by $V$.

We begin with the following relations in $Q_{g}$.

Lemma 7.2. Fix $g \geq 1$, and let $\left\{s_{1}, s_{2}, s_{3}\right\} \subset H_{L}$ be a unimodular set such that $i\left(s_{1}, s_{3}\right)=i\left(s_{2}, s_{3}\right)=0$ and $-1 \leq i\left(s_{1}, s_{2}\right) \leq 1$. Then for all $v \in H_{L}$ we have the following two relations.

$$
\begin{aligned}
X\left(v, s_{2}, s_{3}\right) & =X\left(v+s_{1}, s_{2}, s_{3}\right)+X\left(v, s_{1}, s_{3}\right)-X\left(v+s_{2}, s_{1}, s_{3}\right), \\
X\left(v-s_{1}, s_{2}, s_{3}\right) & =X\left(v, s_{2}, s_{3}\right)+X\left(v-s_{1}, s_{1}, s_{3}\right)-X\left(v-s_{1}+s_{2}, s_{1}, s_{3}\right) .
\end{aligned}
$$

Proof. The first relation follows from the fact that

$$
X\left(v, s_{1}+s_{2}, s_{3}\right)=X\left(v, s_{1}, s_{3}\right)+X\left(v+s_{1}, s_{2}, s_{3}\right) \quad \text { and } \quad X\left(v, s_{1}+s_{2}, s_{3}\right)=X\left(v, s_{2}, s_{3}\right)+X\left(v+s_{2}, s_{1}, s_{3}\right) .
$$

The second follows from the first via the substitution $v \mapsto v-s_{1}$. 
We next show that $\langle V\rangle$ contains several other classes of elements. Define

$$
\begin{gathered}
V_{3}=\left\{X\left(v, s_{1}+e s_{2}, s_{3}+e^{\prime} s_{4}\right) \mid v \in H_{L}, s_{1}, s_{2}, s_{3}, s_{4} \in B \text { distinct, } e, e^{\prime} \in\{-1,1\},\right. \\
\left.i\left(s_{1}, s_{3}\right)= \pm 1, i\left(s_{1}+e s_{2}, s_{3}+e^{\prime} s_{4}\right)=0\right\}, \\
V_{4}=\left\{X\left(v, s_{1}+e s_{2}, s_{1}+e s_{2}+e^{\prime} s_{3}\right) \mid v \in H_{L}, s_{1}, s_{2}, s_{3} \in B \text { distinct, } e, e^{\prime} \in\{-1,1\},\right. \\
\left.i\left(s_{1}, s_{2}\right)= \pm 1, i\left(s_{1}, s_{3}\right)=i\left(s_{2}, s_{3}\right)=0\right\} .
\end{gathered}
$$

We then have the following.

Lemma 7.3. For $g \geq 1$, we have $V_{3}, V_{4} \subset\langle V\rangle$.

Proof. For $x, y \in Q_{g}$, write $x \equiv y$ if $x$ and $y$ are equal modulo $\left\langle V_{1}, V_{2}\right\rangle$. First consider $X\left(v, w_{1}, w_{2}\right) \in V_{3}$. Our goal is to show that $X\left(v, w_{1}, w_{2}\right) \equiv 0$. For concreteness, we will do the case $X\left(v, w_{1}, w_{2}\right)=X\left(v, a_{i}+b_{j}, b_{i}+a_{j}\right)$ for some $1 \leq i, j \leq g$ with $i \neq j$; the other cases are similar.

Observe first that Lemma 7.2 implies that

$$
X\left(v, b_{i}+a_{j}, a_{i}+b_{j}\right)=X\left(v+a_{i}, b_{i}+a_{j}, a_{i}+b_{j}\right)+X\left(v, a_{i}, a_{i}+b_{j}\right)-X\left(v+b_{i}+a_{j}, a_{i}, a_{i}+b_{j}\right) .
$$

Since $X\left(v, a_{i}, a_{i}+b_{j}\right), X\left(v+b_{i}+a_{j}, a_{i}, a_{i}+b_{j}\right) \in V_{2}$, we deduce that $X\left(v, a_{i}+b_{j}, b_{i}+a_{j}\right) \equiv X\left(v+a_{i}, a_{i}+\right.$ $\left.b_{j}, b_{i}+a_{j}\right)$. In a similar manner, we have $X\left(v+a_{i}, a_{i}+b_{j}, b_{i}+a_{j}\right) \equiv X\left(v+\left(a_{i}+b_{j}\right), a_{i}+b_{j}, b_{i}+a_{j}\right)$. Iterating this, we obtain $X\left(v, a_{i}+b_{j}, b_{i}+a_{j}\right) \equiv X\left(v+k\left(a_{i}+b_{j}\right), a_{i}+b_{j}, b_{i}+a_{j}\right)$ for all $k \in \mathbb{Z}$. But this implies that

$$
X\left(v, a_{i}+b_{j}, b_{i}+a_{j}\right) \equiv \frac{1}{L} \sum_{k=0}^{L-1} X\left(v+k\left(a_{i}+b_{j}\right), a_{i}+b_{j}, b_{i}+a_{j}\right)=0
$$

as desired.

Now consider $X\left(v^{\prime}, w_{1}^{\prime}, w_{2}^{\prime}\right) \in V_{4}$. We will show that $X\left(v^{\prime}, w_{1}^{\prime}, w_{2}^{\prime}\right)$ can be written as a linear combination of elements of $V_{1} \cup V_{2} \cup V_{3}$. For concreteness, we will do the case $X\left(v^{\prime}, w_{1}^{\prime}, w_{2}^{\prime}\right)=X\left(v, a_{i}+b_{i}, a_{i}+b_{i}+a_{j}\right)$ for some $1 \leq i, j \leq g$ with $i \neq j$; the other cases are similar. In this case, we have

$$
\begin{aligned}
X\left(v, a_{i}+b_{i}, a_{i}+b_{i}+a_{j}\right)= & X\left(v,\left(a_{i}+b_{j}\right)+\left(b_{i}-b_{j}\right), a_{i}+b_{i}+a_{j}\right) \\
= & X\left(v, a_{i}+b_{j}, a_{i}+b_{i}+a_{j}\right)+X\left(v+a_{i}+b_{j}, b_{i}-b_{j}, a_{i}+b_{i}+a_{j}\right) \\
= & X\left(v, a_{i}+b_{j}, a_{i}\right)+X\left(v+a_{i}, a_{i}+b_{j}, b_{i}+a_{j}\right) \\
& +X\left(v+a_{i}+b_{j}, b_{i}-b_{j}, b_{i}\right)+X\left(v+a_{i}+b_{j}+b_{i}, b_{i}-b_{j}, a_{i}+a_{j}\right),
\end{aligned}
$$

as desired.

Next, say that $v \in H_{L}$ is a simple element of length at most $k$ if it can be written as

$$
v=\sum_{i=1}^{g}\left(c_{i} a_{i}+d_{i} b_{i}\right)
$$

for some $c_{i}, d_{i} \in\{-1,0,1\}$ such that at most $k$ of the $c_{i}$ and $d_{i}$ are nonzero. Define

$$
\begin{array}{r}
V^{\prime}=\left\{X\left(v, w_{1}, w_{2}\right) \mid v, w_{1}, w_{1} \in H_{L},\left\{w_{1}, w_{2}\right\}\right. \text { is isotropic and unimodular, and } \\
w_{1} \text { is a simple element of length at most } 3
\end{array}
$$

We then have the following

Lemma 7.4. For $g \geq 1$, we have $V^{\prime} \subset\langle V\rangle$.

Proof. An easy case-by-case check shows that one can use the "bilinearity relations" (relations 1, 2, and 4 in Lemma 6.6) to express every element of $V^{\prime}$ as a linear combination of elements of $V \cup V_{3} \cup V_{4}$, and thus via Lemma 7.3 as a linear combination of elements of $V$. 


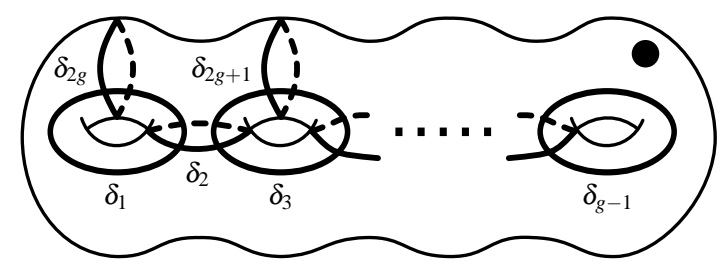

Figure 8: Generators for $\operatorname{Mod}_{g}^{1}$

We finally prove Lemma 7.1.

Proof of Lemma 7.1. The mapping class group $\operatorname{Mod}_{g}^{1}$ acts on both $H_{L}$ and $Q_{g}$. The action on $H_{L}$ is transitive on pairs $\left\{w_{1}, w_{2}\right\}$ of vectors that are isotropic and unimodular. It follows that the $\operatorname{Mod}_{g}^{1}$-orbit of the set $V$ contains every element $X\left(v, w_{1}, w_{2}\right)$. Lemma 6.5 says that these generate $Q_{g}$, so we conclude that it is enough to show that $\langle V\rangle$ is invariant under $\operatorname{Mod}_{g}^{1}$. Let $\left\{\delta_{1}, \ldots, \delta_{2 g+1}\right\}$ be the simple closed curves depicted in Figure 8. The set

$$
S=\left\{T_{\delta_{i}} \mid 1 \leq i \leq 2 g+1\right\}
$$

then generates $\operatorname{Mod}_{g}^{1}$ (see [8]). It is enough to prove that $s \cdot X\left(v, w_{1}, w_{2}\right) \in\langle V\rangle$ for $s \in S^{ \pm 1}$ and $X\left(v, w_{1}, w_{2}\right) \in V$. However, it is easy to see that $s\left(w_{1}\right)$ is a simple element of length at most 3 , so $s \cdot X\left(v, w_{1}, w_{2}\right) \in V^{\prime}$ and the desired result follows from Lemma 7.4.

\section{2 $\psi$ is an isomorphism}

In this section, we prove Lemma 5.4, which we recall asserts that for $g \geq 3$, the map $\psi: Q_{g} \rightarrow I_{g}$ is an isomorphism. Our proof is lengthy, but the basic idea is as follows.

- Using the relations in $Q_{g}$, we will show that the set $\langle V\rangle$ is generated by a set containing $\operatorname{dim} \mathbb{Q}\left[H_{L}\right]-1$ elements.

- By carefully examining the image of $\psi$, we will show that $\mathbb{Q}\left[H_{L}\right]=\psi(\langle V\rangle)+\langle\llbracket 0 \rrbracket\rangle$.

A simple dimension count will then establish the lemma.

Some parts of our proof will be by induction on $g$. We will thus need notation for $\mathbb{Q}\left[H_{L}\right]$ which takes $g$ into account, so define $\mathscr{B}_{g}=\mathbb{Q}\left[\mathrm{H}_{1}\left(\Sigma_{g} ; \mathbb{Z} / L\right)\right]$.

To make the calculations a bit more palatable, we will break this down into several steps. We first determine what $\psi$ does to $V_{1}$.

Lemma 7.5. Fix $g \geq 1$. Set $\mathscr{B}_{g}^{1}=\left\langle\left\{\llbracket c a_{i}+d b_{i} \rrbracket \mid c, d \in \mathbb{Z} / L, 1 \leq i \leq g\right\}\right\rangle$. Then the map $\left.\psi\right|_{\left\langle V_{1}\right\rangle}$ is injective and $\mathscr{B}_{g}=\psi\left(\left\langle V_{1}\right\rangle\right) \oplus \mathscr{B}_{g}^{1}$.

Proof. The proof will by induction on $g$. For the base case $g=1$, the set $V_{1}$ is empty and the assertion is trivial. Assume now that $g \geq 2$ and that the lemma is true for all smaller $g$. Define

$$
\begin{aligned}
V_{1}^{I} & =\left\{X\left(v, s_{1}, s_{2}\right) \in V_{1} \mid s_{1}, s_{2} \notin\left\{a_{g}, b_{g}\right\}\right\}, \\
V_{1}^{A} & =\left\{X\left(v, a_{g}, s\right) \mid X\left(v, a_{g}, s\right) \in V_{1}\right\}, \\
V_{1}^{B} & =\left\{X\left(v, b_{g}, s\right) \mid X\left(v, b_{g}, s\right) \in V_{1}\right\},
\end{aligned}
$$

so $V=V_{1}^{I} \cup V_{1}^{A} \cup V_{1}^{B}$. The proof will consist of three steps.

Step 1. Set $\mathscr{B}_{g}^{2}=\left\langle\left\{\llbracket c a_{i}+d b_{i}+e a_{g}+f b_{g} \rrbracket \mid c, d, e, f \in \mathbb{Z} / L, 1 \leq i \leq g-1\right\}\right\rangle$. Then the map $\left.\psi\right|_{\left\langle V_{1}^{I}\right\rangle}$ is injective and $\mathscr{B}_{g}=\psi\left(\left\langle V_{1}^{I}\right\rangle\right) \oplus \mathscr{B}_{g}^{2}$. 
For $e, f \in \mathbb{Z} / L$, define

$$
\begin{aligned}
\mathscr{B}_{g}(e, f) & =\left\langle\left\{\llbracket v+e a_{g}+f b_{g} \rrbracket \mid v \in\left\langle a_{1}, b_{1}, \ldots, a_{g-1}, b_{g-1}\right\rangle\right\}\right\rangle, \\
\mathscr{B}_{g}^{2}(e, f) & =\left\langle\left\{\llbracket c a_{i}+d b_{i}+e a_{g}+f b_{g} \rrbracket \mid c, d \in \mathbb{Z} / L, 1 \leq i \leq g-1\right\}\right\rangle, \\
V_{1}^{I}(e, f) & =\left\{X\left(v+e a_{g}+f b_{g}, s_{1}, s_{2}\right) \in V_{1} \mid s_{1}, s_{2} \notin\left\{a_{g}, b_{g}\right\}, v \in\left\langle a_{1}, b_{1}, \ldots, a_{g-1}, b_{g-1}\right\rangle\right\} .
\end{aligned}
$$

Observe that

$$
\mathscr{B}_{g}=\bigoplus_{e, f \in \mathbb{Z} / L} \mathscr{B}_{g}(e, f) \quad \text { and } \quad \mathscr{B}_{g}^{2}=\bigoplus_{e, f \in \mathbb{Z} / L} \mathscr{B}_{g}^{2}(e, f) \quad \text { and } \quad V_{1}^{I}=\bigsqcup_{e, f \in \mathbb{Z} / L} V_{1}^{I}(e, f)
$$

Moreover, for all $e, f \in \mathbb{Z} / L$ we have $\psi\left(V_{1}^{I}(e, f)\right) \subset \mathscr{B}_{g}(e, f)$. We conclude that it is enough to prove that $\left.\psi\right|_{\psi\left(\left\langle V_{1}^{I}(e, f)\right\rangle\right)}$ is injective and $\mathscr{B}_{g}(e, f)=\psi\left(\left\langle V_{1}^{I}(e, f)\right\rangle\right) \oplus \mathscr{B}_{g}^{2}(e, f)$ for all $e, f \in \mathbb{Z} / L$.

Consider $e, f \in \mathbb{Z} / L$. The map $\llbracket v \rrbracket \mapsto \llbracket v+e a_{g}+f b_{g} \rrbracket$ induces an isomorphism $\rho: \mathscr{B}_{g-1} \rightarrow \mathscr{B}_{g}(e, f)$ that restricts to an isomorphism $\mathscr{B}_{g-1}^{1} \cong \mathscr{B}_{g}^{2}(e, f)$. Let $V_{1, g-1} \subset Q_{g-1}$ be the $(g-1)$-dimensional analogue of $V_{1}$ and $\psi_{g-1}: Q_{g-1} \rightarrow \mathscr{B}_{g-1}$ be the $(g-1)$-dimensional analogue of $\psi$. The map $X\left(v, w_{1}, w_{2}\right) \mapsto X\left(v+e a_{g}+\right.$ $\left.f b_{g}, w_{1}, w_{2}\right)$ induces a homomorphism $Q_{g-1} \rightarrow Q_{g}$ that restricts to a surjection $\rho^{\prime}:\left\langle V_{1, g-1}\right\rangle \rightarrow\left\langle V_{1}^{I}(e, f)\right\rangle$. Observe that the diagram

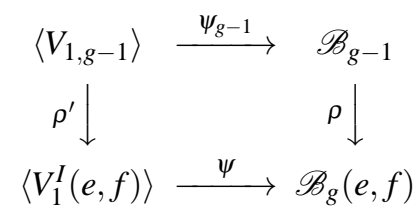

commutes. By the induction hypothesis, $\left.\psi_{g-1}\right|_{\left\langle V_{1, g-1}\right\rangle}$ is injective and $\mathscr{B}_{g-1}=\psi_{g-1}\left(\left\langle V_{1, g-1}\right\rangle\right) \oplus \mathscr{B}_{g-1}^{1}$. We conclude that $\rho^{\prime}$ is injective and thus an isomorphism. Moreover, $\left.\psi\right|_{\left\langle V_{1}^{I}(e, f)\right\rangle}$ is injective and $\mathscr{B}_{g}(e, f)=$ $\psi\left(\left\langle V_{1}^{I}(e, f)\right\rangle\right) \oplus \mathscr{B}_{g}^{2}(e, f)$, as desired.

Step 2. Set $\mathscr{B}_{g}^{3}=\left\langle\left\{\llbracket c a_{i}+d b_{i}+f b_{g} \rrbracket\right.\right.$, $\left.\left.\llbracket e a_{g}+f b_{g} \rrbracket \mid c, d, e, f \in \mathbb{Z} / L, 1 \leq i \leq g-1\right\}\right\rangle$. Then the map $\left.\psi\right|_{\left\langle V_{1}^{I}, V_{1}^{A}\right\rangle}$ is injective and $\mathscr{B}_{g}=\psi\left(\left\langle V_{1}^{I}, V_{1}^{A}\right\rangle\right) \oplus \mathscr{B}_{g}^{3}$.

Define

$$
V_{1}^{A, 1}=\left\{X\left(c a_{i}+d b_{i}+e a_{g}+f b_{g}, a_{g}, s\right) \mid 1 \leq i \leq g-1, c, d, e, f \in \mathbb{Z} / L, s \in\left\{a_{i}, b_{i}\right\}\right\} \subset V_{1}^{A} .
$$

We claim that $\left\langle V_{1}^{I}, V_{1}^{A}\right\rangle=\left\langle V_{1}^{I}, V_{1}^{A, 1}\right\rangle$. Indeed, consider $X\left(w, a_{g}, s\right) \in V_{1}^{A}$. By Lemma 7.2, for any $s^{\prime} \in$ $\left\{a_{1}, b_{1}, \ldots, a_{g-1}, b_{g-1}\right\}$ with $s^{\prime} \neq s$ and $i\left(s, s^{\prime}\right)=0$, we have

$$
X\left(w-s^{\prime}, a_{g}, s\right)=X\left(w, a_{g}, s\right)+X\left(w-s^{\prime}, s^{\prime}, s\right)-X\left(w-s^{\prime}+a_{g}, s^{\prime}, s\right) .
$$

Hence modulo $\left\langle V_{1}^{I}\right\rangle$, we have $X\left(w, a_{g}, s\right)$ equal to $X\left(w-s^{\prime}, a_{g}, s\right)$. Iterating this, modulo $\left\langle V_{1}^{I}\right\rangle$ we have $X\left(w, a_{g}, s\right)$ equal to an element of $V_{1}^{A, 1}$, as desired.

For $x \in \mathbb{Z} / L$, we will denote by $|x|$ the unique integer representing $x$ with $0 \leq|x|<L-1$. Noting that $\psi\left(V_{1}^{A, 1}\right) \subset \mathscr{B}_{g}^{2}$, we claim that $\mathscr{B}_{g}^{2}=\psi\left(\left\langle V_{1}^{A, 1}\right\rangle\right)+\mathscr{B}_{g}^{3}$. Indeed, assume that there is some $\llbracket c a_{i}+d b_{i}+e a_{g}+$ $f b_{g} \rrbracket \in \mathscr{B}_{g}^{2}$ that is not in $\psi\left(\left\langle V_{1}^{A, 1}\right\rangle\right)+\mathscr{B}_{g}^{3}$. Choose $\llbracket c a_{i}+d b_{i}+e a_{g}+f b_{g} \rrbracket$ such that $|c|+|d|+|e|$ is minimal among elements with this property. By assumption we must have $|e|$ and one of $|c|$ or $|d|$ (say $|c|$ ) nonzero. Setting $w^{\prime}=c a_{i}+d b_{i}+e a_{g}+f b_{g}$, we then have $X\left(w^{\prime}-a_{i}-a_{g}, a_{g}, a_{i}\right) \in V_{1}^{A, 1}$ and

$$
\llbracket w^{\prime} \rrbracket-\psi\left(X\left(w^{\prime}-a_{i}-a_{g}, a_{g}, a_{i}\right)\right)=\llbracket w^{\prime}-a_{i} \rrbracket+\llbracket w^{\prime}-a_{g} \rrbracket-\llbracket w^{\prime}-a_{i}-a_{g} \rrbracket .
$$

We conclude that one of $\llbracket w^{\prime}-a_{i} \rrbracket$, $w^{\prime}-a_{g} \rrbracket$, or $\llbracket w^{\prime}-a_{i}-a_{g} \rrbracket$ is not in $\psi\left(\left\langle V_{1}^{A, 1}\right\rangle\right)+\mathscr{B}_{g}^{3}$, contradicting the minimality of $|c|+|d|+|e|$. 
Now define

$$
\begin{aligned}
& V_{1}^{A, 2}=\{\left.X\left(c a_{i}+d b_{i}+e a_{g}+f b_{g}, a_{g}, a_{i}\right) \mid 1 \leq i \leq g-1, c, d, e, f \in \mathbb{Z} / L\right\} \\
& \cup\left\{X\left(d b_{i}+e a_{g}+f b_{g}, a_{g}, b_{i}\right) \mid 1 \leq i \leq g-1, d, e, f \in \mathbb{Z} / L\right\} \subset V_{1}^{A, 1}, \\
& V_{1}^{A, 3}=\left\{X\left(v, a_{g}, s\right) \in V_{1}^{A, 2} \mid \text { the } s \text { and } a_{g} \text {-coordinates of } v \text { do not equal } L-1\right\} \subset V_{1}^{A, 2} .
\end{aligned}
$$

We will prove that $\left\langle V_{1}^{A, 3}\right\rangle=\left\langle V_{1}^{A, 1}\right\rangle$. Using the third relation in Lemma 6.6, we see that $\left\langle V_{1}^{A, 3}\right\rangle=\left\langle V_{1}^{A, 2}\right\rangle$. It is thus enough to prove that $\left\langle V_{1}^{A, 1}\right\rangle=\left\langle V_{1}^{A, 2}\right\rangle$. An element of $V_{1}^{A, 1} \backslash V_{1}^{A, 2}$ is of the form $X\left(w^{\prime \prime}, a_{g}, b_{i}\right)$. Lemma 7.2 says that

$$
X\left(w^{\prime \prime}-a_{i}, b_{i}, a_{g}\right)=X\left(w^{\prime \prime}, b_{i}, a_{g}\right)+X\left(w^{\prime \prime}-a_{i}, a_{i}, a_{g}\right)-X\left(w^{\prime \prime}-a_{i}+b_{i}, a_{i}, a_{g}\right) .
$$

Iterating this, we conclude that modulo $\left\langle V_{1}^{A, 2}\right\rangle$, we have $X\left(w^{\prime \prime}, a_{g}, b_{i}\right)$ equal to an element of $V_{1}^{A, 2}$, as desired.

We deduce from the above two paragraphs that $\mathscr{B}_{g}^{2}=\psi\left(\left\langle V_{1}^{A, 3}\right\rangle\right)+\mathscr{B}_{g}^{3}$. Since $V_{1}^{A, 3}$ contains

$$
\begin{aligned}
(g-1)\left(L^{2}(L-1)^{2}+L(L-1)^{2}\right) & =\left((g-1)\left(L^{2}-1\right) L^{2}+L^{2}\right)-\left((g-1)\left(L^{2}-1\right) L+L^{2}\right) \\
& =\operatorname{dim}\left(\mathscr{B}_{g}^{2}\right)-\operatorname{dim}\left(\mathscr{B}_{g}^{3}\right)
\end{aligned}
$$

elements, we obtain that $\left.\psi\right|_{\left\langle V_{1}^{A, 3}\right\rangle}$ is injective and $\mathscr{B}_{g}^{2}=\psi\left(\left\langle V_{1}^{A, 3}\right\rangle\right) \oplus \mathscr{B}_{g}^{3}$. By Step 1, the fact that $\left\langle V_{1}^{I}, V_{1}^{A}\right\rangle=$ $\left\langle V_{1}^{I}, V_{1}^{A, 1}\right\rangle$, and the fact that $\left\langle V_{1}^{A, 3}\right\rangle=\left\langle V_{1}^{A, 1}\right\rangle$, we conclude that $\left.\psi\right|_{\left\langle V_{1}^{I}, V_{1}^{A}\right\rangle}$ is injective and $\mathscr{B}_{g}=\psi\left(\left\langle V_{1}^{I}, V_{1}^{A}\right\rangle\right) \oplus$ $\mathscr{B}_{g}^{3}$, as desired.

Step 3. Recall that $\mathscr{B}_{g}^{1}=\left\langle\left\{\llbracket c a_{i}+d b_{i} \rrbracket \mid c, d \in \mathbb{Z} / L, 1 \leq i \leq g\right\}\right\rangle$. The map $\left.\psi\right|_{\left\langle V_{1}^{I}, V_{1}^{A}, V_{1}^{B}\right\rangle}$ is injective and $\mathscr{B}_{g}=$ $\psi\left(\left\langle V_{1}^{I}, V_{1}^{A}, V_{1}^{B}\right\rangle\right) \oplus \mathscr{B}_{g}^{1}$.

The argument for this step is very similar to the argument in Step 2, so we only sketch it. Define

$$
\begin{aligned}
V_{1}^{B, 1}=\{ & \left\{\left(c a_{i}+d b_{i}+f b_{g}, b_{g}, s\right) \mid 1 \leq i \leq g-1, c, d, f \in \mathbb{Z} / L, s \in\left\{a_{i}, b_{i}\right\}\right\} \subset V_{1}^{B}, \\
V_{1}^{B, 2}= & \left\{X\left(c a_{i}+d b_{i}+f b_{g}, b_{g}, a_{i}\right) \mid 1 \leq i \leq g-1, c, d, f \in \mathbb{Z} / L\right\} \\
& \cup\left\{X\left(d b_{i}+f b_{g}, b_{g}, b_{i}\right) \mid 1 \leq i \leq g-1, d, f \in \mathbb{Z} / L\right\} \subset V_{1}^{B, 1}, \\
V_{1}^{B, 3}= & \left\{X\left(v, b_{g}, s\right) \in V_{1}^{B, 2} \mid \text { the } s \text { and } b_{g} \text {-coordinates of } v \text { do not equal } L-1\right\} \subset V_{1}^{B, 2} .
\end{aligned}
$$

Noting that $\psi\left(V_{1}^{B, 1}\right) \subset \mathscr{B}_{g}^{3}$, arguments similar to those in Step 2 show that $\left\langle V_{1}^{I}, V_{1}^{A}, V_{1}^{B}\right\rangle=\left\langle V_{1}^{I}, V_{1}^{A}, V_{1}^{B, 1}\right\rangle$, that $\mathscr{B}_{g}^{3}=\psi\left(\left\langle V_{1}^{B, 1}\right\rangle\right)+\mathscr{B}_{g}^{1}$, that $\left\langle V_{1}^{B, 1}\right\rangle=\left\langle V_{1}^{B, 2}\right\rangle$, and that $\left\langle V_{1}^{B, 2}\right\rangle=\left\langle V_{1}^{B, 3}\right\rangle$.

We deduce that $\mathscr{B}_{g}^{3}=\psi\left(\left\langle V_{1}^{B, 3}\right\rangle\right)+\mathscr{B}_{g}^{1}$. Since $V_{1}^{B, 3}$ contains

$$
\begin{aligned}
(g-1)\left((L-1)^{2} L+(L-1)^{2}\right) & =\left((g-1)\left(L^{2}-1\right) L+L^{2}\right)-\left(g\left(L^{2}-1\right)+1\right) \\
& =\operatorname{dim}\left(\mathscr{B}_{g}^{3}\right)-\operatorname{dim}\left(\mathscr{B}_{g}^{1}\right)
\end{aligned}
$$

elements, we obtain that $\left.\psi\right|_{\left\langle V_{1}^{B, 3}\right\rangle}$ is injective and $\mathscr{B}_{g}^{3}=\psi\left(\left\langle V_{1}^{B, 3}\right\rangle\right) \oplus \mathscr{B}_{g}^{1}$. By Step 2 and the identities

$$
\left\langle V_{1}^{I}, V_{1}^{A}, V_{1}^{B}\right\rangle=\left\langle V_{1}^{I}, V_{1}^{A}, V_{1}^{B, 1}\right\rangle \text { and }\left\langle V_{1}^{B, 3}\right\rangle=\left\langle V_{1}^{B_{1}}\right\rangle,
$$

we conclude that $\left.\psi\right|_{\left\langle V_{1}^{I}, V_{1}^{A}, V_{1}^{B}\right\rangle}$ is injective and $\mathscr{B}_{g}=\psi\left(\left\langle V_{1}^{I}, V_{1}^{A}, V_{1}^{B}\right\rangle\right) \oplus \mathscr{B}_{g}^{1}$, as desired.

Our final lemma is a further relation in $Q_{g}$.

Lemma 7.6. Let $\left\{a_{1}^{\prime}, b_{1}^{\prime}, a_{2}^{\prime}, b_{2}^{\prime}\right\}$ be a unimodular subset of $\mathrm{H}_{1}\left(\Sigma_{g} ; \mathbb{Z} / L\right)$ with $i\left(a_{1}^{\prime}, b_{1}^{\prime}\right)=i\left(a_{2}^{\prime}, b_{2}^{\prime}\right)=1$ and $i\left(a_{1}^{\prime}, a_{2}^{\prime}\right)=i\left(a_{1}^{\prime}, b_{2}^{\prime}\right)=i\left(b_{1}^{\prime}, a_{2}^{\prime}\right)=i\left(b_{1}^{\prime}, b_{2}^{\prime}\right)=0$. Then for all $v \in \mathrm{H}_{1}\left(\Sigma_{g} ; \mathbb{Z} / L\right)$ we have

$$
\begin{aligned}
& X\left(v, a_{1}^{\prime}, a_{2}^{\prime}\right)-X\left(v+b_{1}^{\prime}, a_{1}^{\prime}, a_{2}^{\prime}\right)-X\left(v+b_{2}^{\prime}, a_{1}^{\prime}, a_{2}^{\prime}\right)+X\left(v+b_{1}^{\prime}+b_{2}^{\prime}, a_{1}^{\prime}, a_{2}^{\prime}\right) \\
& \quad=X\left(v, b_{1}^{\prime}, b_{2}^{\prime}\right)-X\left(v+a_{1}^{\prime}, b_{1}^{\prime}, b_{2}^{\prime}\right)-X\left(v+a_{2}^{\prime}, b_{1}^{\prime}, b_{2}^{\prime}\right)+X\left(v+a_{1}^{\prime}+a_{2}^{\prime}, b_{1}^{\prime}, b_{2}^{\prime}\right)
\end{aligned}
$$


Proof. The group $\mathrm{Sp}_{2 g}(\mathbb{Z})$ acts on $Q_{g}$, and there exists some $f \in \operatorname{Sp}_{2 g}(\mathbb{Z})$ such that $f\left(a_{i}^{\prime}\right)=a_{i}$ and $f\left(b_{i}^{\prime}\right)=b_{i}$ for $i=1,2$. We can therefore assume that $a_{i}^{\prime}=a_{i}$ and $b_{i}^{\prime}=b_{i}$ for $i=1,2$. But an easy calculation shows that $\psi$ takes both sides of our relation to the same element of $\mathscr{B}_{g}$, so the lemma follows from Lemma 7.5.

We can now prove Lemma 5.4.

Proof of Lemma 5.4. Define $Q_{g}^{\prime}=Q_{g} /\left\langle V_{1}\right\rangle$ and $\mathscr{B}_{g}^{\prime}=\mathscr{B}_{g} / \psi\left(\left\langle V_{1}\right\rangle\right)$. We have an induced map $\psi^{\prime}: Q_{g}^{\prime} \rightarrow \mathscr{B}_{g}^{\prime}$. Using the direct sum decomposition of Lemma 7.5, we will identify $\mathscr{B}_{g}^{\prime}$ with the subspace

$$
\left\langle\left\{\llbracket c a_{i}+d b_{i} \rrbracket \mid c, d \in \mathbb{Z} / L, 1 \leq i \leq g\right\}\right\rangle
$$

of $\mathscr{B}_{g}$. Letting $V_{2}^{\prime} \subset Q_{g}^{\prime}$ be the image of $V_{2} \subset Q_{g}$, Lemmas 7.1 and 7.5 say that it is enough to prove that $\left.\psi^{\prime}\right|_{\left\langle V_{2}^{\prime}\right\rangle}$ is injective and $\mathscr{B}_{g}^{\prime}=\psi^{\prime}\left(\left\langle V_{2}^{\prime}\right\rangle\right) \oplus\langle\llbracket 0 \rrbracket\rangle$.

Let $\phi: Q_{g} \rightarrow Q_{g}^{\prime}$ be the projection. The proof will require seven claims. It follows the same pattern as Steps 2 and 3 of the proof of Lemma 7.5. In Claims 1-3 and 5-6, we will obtain a "minimal" size generating set for $\left\langle V_{2}^{\prime}\right\rangle$. In Claims 4 and 7, we will show that $\mathscr{B}_{g}^{\prime}=\psi^{\prime}\left(\left\langle V_{2}^{\prime}\right\rangle\right)+\langle\llbracket 0 \rrbracket\rangle$. A dimension count will then establish the lemma.

Claim 1. Let $s, s_{1}, s_{2} \in B$ satisfy $s \neq s_{1}, s_{2}$ and $i\left(s, s_{1}\right)=i\left(s, s_{2}\right)=0$. Then for all $v \in \mathrm{H}_{1}\left(\Sigma_{g} ; \mathbb{Z} / L\right)$ and $e_{1}, e_{2} \in\{-1,1\}$, we have $\phi\left(X\left(v, s, s+e_{1} s_{1}\right)\right)=\phi\left(X\left(v, s, s+e_{2} s_{2}\right)\right)$.

Proof of Claim. For $1 \leq i \leq 2$, using the second relation in Lemma 6.6, we get that

$$
X\left(v+e_{1} s_{1}+s, e_{2} s_{2}, s\right), X\left(v+e_{2} s_{2}+s, e_{1} s_{1}, s\right) \in\left\langle V_{1}\right\rangle .
$$

Hence

$$
\phi\left(X\left(v, e_{1} s_{1}+e_{2} s_{2}+s, s\right)\right)=\phi\left(X\left(v, e_{1} s_{1}+s, s\right)+X\left(v+e_{1} s_{1}+s, e_{2} s_{2}, s\right)\right)=\phi\left(X\left(v, s, s+e_{1} s_{1}\right)\right)
$$

and

$$
\phi\left(X\left(v, e_{1} s_{1}+e_{2} s_{2}+s, s\right)\right)=\phi\left(X\left(v, e_{2} s_{2}+s, s\right)+X\left(v+e_{2} s_{2}+s, e_{1} s_{1}, s\right)\right)=\phi\left(X\left(v, s, s+e_{2} s_{2}\right)\right) .
$$

The claim follows.

In light of Claim 1, we will denote by $Y(v, s)$ the image in $V_{2}^{\prime}$ of $X\left(v, s, s+e^{\prime} s^{\prime}\right)$, where $e^{\prime} \in\{-1,1\}$ and $s^{\prime} \in B$ are arbitrary elements such that $X\left(v, s, s+e^{\prime} s^{\prime}\right) \in V_{2}$.

Claim 2. Consider $Y(v, s) \in V_{2}^{\prime}$. Pick $1 \leq i \leq g$ such that $s \in\left\{a_{i}, b_{i}\right\}$. Write $v=v_{1}+v_{2}$ with $v_{1} \in\left\langle a_{i}, b_{i}\right\rangle$ and $v_{2} \in\left\langle\left\{a_{j}, b_{j} \mid j \neq i\right\}\right\rangle$. Then $Y(v, s)=Y\left(v_{1}, s\right)$.

Proof of Claim. Consider $s^{\prime} \in\left\{a_{j}, b_{j} \mid j \neq i\right\}$. It is enough to show that $Y\left(v-s^{\prime}, s\right)=Y(v, s)$. Pick $s^{\prime \prime} \in$ $\left\{a_{j}, b_{j} \mid j \neq i\right\}$ such that $s^{\prime \prime} \neq s^{\prime}$ and $i\left(s^{\prime}, s^{\prime \prime}\right)=0$ (this uses the fact that $g \geq 3$ ). Observe that $Y(v, s)=$ $\phi\left(X\left(v, s, s+s^{\prime \prime}\right)\right)$ and $Y\left(v-s^{\prime}, s\right)=\phi\left(X\left(v-s^{\prime}, s, s+s^{\prime \prime}\right)\right)$. Lemma 7.2 says that

$$
X\left(v-s^{\prime}, s, s+s^{\prime \prime}\right)=X\left(v, s, s+s^{\prime \prime}\right)+X\left(v-s^{\prime}, s^{\prime}, s+s^{\prime \prime}\right)-X\left(v-s^{\prime}+s, s^{\prime}, s+s^{\prime \prime}\right) .
$$

For $w$ equal to $v-s^{\prime}$ or $v-s^{\prime}+s$, we have

$$
X\left(w, s^{\prime}, s+s^{\prime \prime}\right)=X\left(w, s+s^{\prime \prime}, s^{\prime}\right)=X\left(w, s, s^{\prime}\right)+X\left(w+s, s^{\prime \prime}, s^{\prime}\right) \in\left\langle V_{1}\right\rangle .
$$

Applying $\phi$ to both sides of (9), we thus obtain that $Y(v, s)=Y\left(v-s^{\prime}, s\right)$, as desired.

Claim 3. For all $1 \leq i \leq g$ and $v \in\left\langle a_{i}, b_{i}\right\rangle$, we have

$$
Y\left(v, a_{i}\right)-2 Y\left(v+b_{i}, a_{i}\right)+Y\left(v+2 b_{i}, a_{i}\right)=Y\left(v, b_{i}\right)-2 Y\left(v+a_{i}, b_{i}\right)+Y\left(v+2 a_{i}, b_{i}\right) .
$$


Proof of Claim. Pick $1 \leq j<k \leq g$ such that $i \neq j, k$ (this uses the fact that $g \geq 3$ ). Lemma 7.6 applied with $\left(a_{1}^{\prime}, b_{1}^{\prime}, a_{2}^{\prime}, b_{2}^{\prime}\right)=\left(a_{i}+a_{k}, b_{i}-b_{j}, a_{i}+a_{j}, b_{i}-b_{k}\right)$ says that

$$
\begin{aligned}
& X\left(v, a_{i}+a_{k}, a_{i}+a_{j}\right)-X\left(v+b_{i}-b_{j}, a_{i}+a_{k}, a_{i}+a_{j}\right)-X\left(v+b_{i}-b_{k}, a_{i}+a_{k}, a_{i}+a_{j}\right) \\
& +X\left(v+b_{i}-b_{j}+b_{i}-b_{k}, a_{i}+a_{k}, a_{i}+a_{j}\right) \\
= & X\left(v, b_{i}-b_{j}, b_{i}-b_{k}\right)-X\left(v+a_{i}+a_{k}, b_{i}-b_{j}, b_{i}-b_{k}\right)-X\left(v+a_{i}+a_{j}, b_{i}-b_{j}, b_{i}-b_{k}\right) \\
& +X\left(v+a_{i}+a_{k}+a_{i}+a_{j}, b_{i}-b_{j}, b_{i}-b_{k}\right) .
\end{aligned}
$$

Since $X\left(v+a_{i}, a_{k}, a_{i}+a_{j}\right) \in\left\langle V_{1}\right\rangle$, we have that

$$
\phi\left(X\left(v, a_{i}+a_{k}, a_{i}+a_{j}\right)\right)=\phi\left(X\left(v, a_{i}, a_{i}+a_{j}\right)+X\left(v+a_{i}, a_{k}, a_{i}+a_{j}\right)\right)=Y\left(v, a_{i}\right) .
$$

Similarly, we have $\phi\left(X\left(v+b_{i}-b_{j}, a_{i}+a_{k}, a_{i}+a_{j}\right)\right)=Y\left(v+b_{i}-b_{j}, a_{i}\right)$. By Claim 2, this equals $Y\left(v+b_{i}, a_{i}\right)$. Continuing in this manner, we deduce that $\phi$ maps (10) to the desired relation between the $Y(\cdot, \cdot)$.

For the next claim, recall that we are using Lemma 7.5 to identify $\mathscr{B}_{g}^{\prime}=\mathscr{B}_{g} / \psi\left(\left\langle V_{1}\right\rangle\right)$ with the subspace $\left\langle\left\{\llbracket c a_{i}+d b_{i} \rrbracket \mid c, d \in \mathbb{Z} / L, 1 \leq i \leq g\right\}\right\rangle$ of $\mathscr{B}_{g}$.

Claim 4. For some $1 \leq i \leq g$, let $s \in\left\{a_{i}, b_{i}\right\}$ and $v \in\left\langle a_{i}, b_{i}\right\rangle$. Then $\psi^{\prime}(Y(v, s))=\llbracket v \rrbracket-2 \llbracket v+s \rrbracket+\llbracket v+2 s \rrbracket$.

Proof of Claim. Let $\rho: \mathscr{B}_{g} \rightarrow \mathscr{B}_{g}^{\prime}$ be the projection. Pick $1 \leq j \leq g$ such that $j \neq i$. Observe that $Y(v, s)=$ $\phi\left(X\left(v, s, s-a_{j}\right)\right)$ and $X\left(v+s-a_{j}, a_{j}, s\right) \in V_{1}$. Thus $\psi^{\prime}(Y(v, s))$ equals

$$
\begin{aligned}
\rho\left(\psi\left(X\left(v, s-a_{j}, s\right)\right)\right)= & \rho\left(\psi\left(X\left(v, s-a_{j}, s\right)+X\left(v+s-a_{j}, a_{j}, s\right)\right)\right) \\
= & \rho\left(\left(\llbracket v \rrbracket-\llbracket v+s-a_{j} \rrbracket-\llbracket v+s \rrbracket+\llbracket v+2 s-a_{j} \rrbracket\right)\right. \\
& \quad+\left(\llbracket v+s-a_{j} \rrbracket-\llbracket v+s \rrbracket-\llbracket v+2 s-a_{j} \rrbracket+\llbracket v+2 s \rrbracket\right) \\
= & \rho(\llbracket v \rrbracket-2 \llbracket v+s \rrbracket+\llbracket v+2 s \rrbracket) .
\end{aligned}
$$

Since $v, v+s, v+2 s \in\left\langle a_{i}, b_{i}\right\rangle$, this equals $\llbracket v \rrbracket-2 \llbracket v+2 \rrbracket+\llbracket v+2 s \rrbracket$, as desired.

For some $1 \leq i \leq g$, consider $s \in\left\{a_{i}, b_{i}\right\}$ and $v \in\left\langle a_{i}, b_{i}\right\rangle$. Making use of Claim 4, an easy induction establishes that for $n \geq 1$, we have

$$
\psi^{\prime}\left(\sum_{k=1}^{n} k \cdot Y(v+(k-1) s, s)\right)=\llbracket v \rrbracket-(n+1) \llbracket v+n s \rrbracket+n \llbracket v+(n+1) s \rrbracket .
$$

In particular, setting $Z(v, s)=\sum_{k=1}^{L} k \cdot Y(v+(k-1) s, s)$, we have

$$
\psi^{\prime}(Z(v, s))=L \llbracket v+s \rrbracket-L \llbracket v \rrbracket .
$$

We now prove the following.

Claim 5. For all $1 \leq i \leq g$ and $v \in\left\langle a_{i}, b_{i}\right\rangle$, we have

$$
Z\left(v, a_{i}\right)-2 Z\left(v+b_{i}, a_{i}\right)+Z\left(v+2 b_{i}, a_{i}\right)=L \cdot Y\left(v+a_{i}, b_{i}\right)-L \cdot Y\left(v, b_{i}\right) .
$$

Proof of Claim. Observe that $Z\left(v, a_{i}\right)-2 Z\left(v+b_{i}, a_{i}\right)+Z\left(v+2 b_{i}, a_{i}\right)$ equals

$$
\sum_{k=1}^{L} k \cdot\left(Y\left(v+(k-1) a_{i}, a_{i}\right)-2 Y\left(v+(k-1) a_{i}+b_{i}, a_{i}\right)+Y\left(v+(k-1) a_{i}+2 b_{i}, a_{i}\right)\right) .
$$

By Claim 3, this equals

$$
\sum_{k=1}^{L} k \cdot\left(Y\left(v+(k-1) a_{i}, b_{i}\right)-2 Y\left(v+k a_{i}, b_{i}\right)+Y\left(v+(k+1) a_{i}, b_{i}\right)\right) .
$$

An argument similar to the argument used to calculate $\psi^{\prime}$ of $Z(\cdot, \cdot)$ then shows that this equals $L \cdot Y(v+$ $\left.a_{i}, b_{i}\right)-L \cdot Y\left(v, b_{i}\right)$, and we are done. 
Claim 6. We have

$$
\begin{gathered}
\left\langle V_{2}^{\prime}\right\rangle=\left\langle\left\{Y\left(c a_{i}+d b_{i}, a_{i}\right) \mid 1 \leq i \leq g, c, d \in \mathbb{Z} / L, c \neq L-1\right\}\right. \\
\left.\cup\left\{Y\left(d b_{i}, b_{i}\right) \mid 1 \leq i \leq g, d \in \mathbb{Z} / L, d \neq L-1\right\}\right\rangle .
\end{gathered}
$$

Proof of Claim. Claim 2 implies that

$$
\left\langle V_{2}^{\prime}\right\rangle=\left\{Y(v, s) \mid v \in\left\langle a_{i}, b_{i}\right\rangle \text { and } s \in\left\{a_{i}, b_{i}\right\} \text { for some } 1 \leq i \leq g\right\}
$$

If $v \in\left\{a_{i}, b_{i}\right\}$ for some $1 \leq i \leq g$, then

$$
Z\left(v, a_{i}\right) \in\left\langle\left\{Y\left(c a_{i}+d b_{i}, a_{i}\right) \mid 1 \leq i \leq g, c, d \in \mathbb{Z} / L\right\}\right\rangle .
$$

We can therefore use the relation in Claim 5 to reduce (12) to

$$
\begin{gathered}
\left\langle V_{2}^{\prime}\right\rangle=\left\langle\left\{Y\left(c a_{i}+d b_{i}, a_{i}\right) \mid 1 \leq i \leq g, c, d \in \mathbb{Z} / L\right\}\right. \\
\left.\cup\left\{Y\left(d b_{i}, b_{i}\right) \mid 1 \leq i \leq g, d \in \mathbb{Z} / L\right\}\right\rangle .
\end{gathered}
$$

Finally, if $v \in\left\langle a_{i}, b_{i}\right\rangle$ and $s \in\left\{a_{i}, b_{i}\right\}$ for some $1 \leq i \leq g$, then from the third relation in Lemma 6.6, we obtain the relation $\sum_{k=0}^{L-1} Y(v+k \cdot s, s)=0$ in $Q_{g}^{\prime}$. This allows us to reduce (13) to the claimed generating set, and we are done.

Claim 7. $\mathscr{B}_{g}^{\prime}=\left\langle\psi^{\prime}\left(V_{2}^{\prime}\right)\right\rangle+\langle\llbracket 0 \rrbracket\rangle$.

Proof of Claim. Consider $c, d \geq 0$ with $(c, d) \neq 0$. With the convention that an empty sum of abelian group elements is the zero element, we can use (11) to get that

$$
\begin{aligned}
\frac{1}{L} \psi^{\prime}\left(\sum_{j=1}^{c} Z\left(j a_{i}, a_{i}\right)+\sum_{k=1}^{d} Z\left(c a_{i}+k b_{i}, b_{i}\right)\right) & =\left(\llbracket c a_{i} \rrbracket-\llbracket 0 \rrbracket\right)+\left(\llbracket c a_{i}+d b_{i} \rrbracket-\llbracket c a_{i} \rrbracket\right) \\
& =\llbracket c a_{i}+d b_{i} \rrbracket-\llbracket 0 \rrbracket .
\end{aligned}
$$

Here the first equality follows from the fact that the indicated sums become telescoping sums after applying $\psi^{\prime}$. The claim follows.

Observe now that the generating set for $\left\langle V_{2}^{\prime}\right\rangle$ given by Claim 6 has

$$
g(L(L-1)+L-1)=g\left(L^{2}-1\right)=\operatorname{dim}\left(\mathscr{B}_{g}^{\prime}\right)-1
$$

elements, so by Claim 7 we have that $\left.\psi^{\prime}\right|_{\left\langle V_{2}^{\prime}\right\rangle}$ is injective and $\mathscr{B}_{g}^{\prime}=\left\langle\psi^{\prime}\left(V_{2}^{\prime}\right)\right\rangle \oplus\langle\llbracket 0 \rrbracket\rangle$, as desired.

\section{$8 \quad$ Killing off $\mathscr{S}_{g}$}

This section is devoted to the proof of Lemma 5.5. The proof itself is contained in $\S 8.2$. This is proceeded by $\S 8.1$, which contains a technical lemma about essentially separate curves.

\subsection{Separating essentially separate curves}

This section is devoted to the proof of the following lemma.

Lemma 8.1. Consider $v, v^{\prime} \in H_{L}$ and $x, y \in K_{g}$ such that $x$ and $y$ are essentially separate and $\left\langle\langle y\rangle \in C_{g}\right.$. There then exists some $n \geq 1$ and $v_{1}, v_{1}^{\prime}, \ldots, v_{n}, v_{n}^{\prime} \in H_{L}$ and $x_{1}, y_{1}, \ldots, x_{n}, y_{n} \in K_{g}$ with the following properties.

1. $\langle\langle x\rangle\rangle^{v} \otimes\langle\langle y\rangle\rangle^{\prime}=\sum_{i=1}^{n}\left\langle\left\langle x_{i}\right\rangle\right\rangle^{v_{i}} \otimes\left\langle\left\langle y_{i}\right\rangle v_{i}^{\prime}\right.$. 


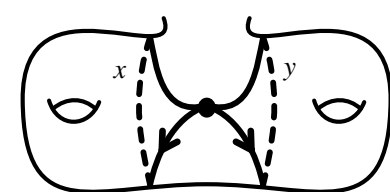

a

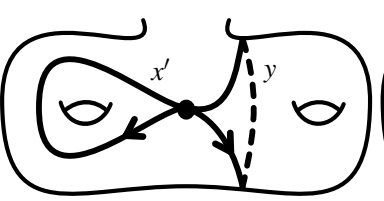

b

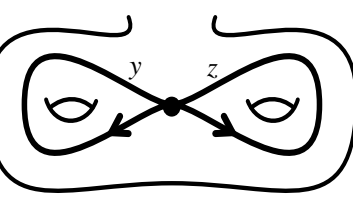

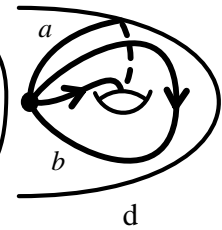

d. $[a, b]$ is $a$

Figure 9: $a, b$. The intersection patterns needed for Lemma 8.1 genus 1 separating curve.

c. A nonseparating figure eight.

2. For all $1 \leq i \leq n$, one of the following two conditions is satisfied.

(a) $\left\{x_{i}, y_{i}\right\}$ has the same unoriented intersection pattern as the curves in Figure 9.a, or

(b) $x_{i}=\left(x_{i}^{\prime}\right)^{L}$, where $\left\{x_{i}^{\prime}, y_{i}\right\}$ has the same unoriented intersection pattern as the curves in Figure 9.b.

Remark. The second condition in the conclusion of Lemma 8.1 implies that $\left\langle\left\langle y_{i}\right\rangle\right\rangle \in C_{g}$.

To prove Lemma 8.1, we will need a definition and a lemma.

Definition. A curve $x \in \pi_{1}\left(\Sigma_{g}\right)$ is a genus $k$ separating curve if it can be realized by a simple closed curve that separates $\Sigma_{g}$ into two pieces, one of which is homeomorphic to $\Sigma_{k, 1}$. Two curves $y, z \subset \pi_{1}\left(\Sigma_{g}\right)$ form a nonseparating figure eight if they have the same unoriented intersection pattern as the curves in Figure 9.c.

Remark. If $a, b \in \pi_{1}\left(\Sigma_{g}\right)$ have the same unoriented intersection pattern as the curves in Figure 9.d, then $[a, b] \in \pi_{1}\left(\Sigma_{g}\right)$ is a genus 1 separating curve.

Lemma 8.2. Let $S \subset \Sigma_{g}$ be a subsurface such that the basepoint lies on $\partial S$ and $\Sigma_{g} \backslash S$ is connected. Define $P_{S}=\operatorname{Im}\left(\pi_{1}(S) \rightarrow \pi_{1}\left(\Sigma_{g}\right)\right)$ and $H_{S}=\operatorname{Im}\left(\mathrm{H}_{1}(S ; \mathbb{Z} / L) \rightarrow H_{L}\right)$. Next, define the following sets.

$$
\begin{aligned}
& U_{1}=\left\{\left\langle\left\langle x^{L}\right\rangle\right| x \in P_{S} \text { is a simple closed nonseparating curve }\right\}, \\
& \left.U_{2}=\{\langle x\rangle\rangle^{v} \mid v \in H_{S}, x \in P_{S} \text { is a genus } 1 \text { separating curve }\right\}, \\
& \left.U_{3}=\{\langle y, z\rangle\rangle^{v} \mid v \in H_{S}, y, z \subset P_{S} \text { form a nonseparating figure eight }\right\} .
\end{aligned}
$$

Then the following hold.

1. $C_{g} \cap \mathrm{H}_{1}\left(K_{g} \cap P_{S} ; \mathbb{Q}\right)$ is spanned by $U_{2} \cup U_{3}$ and $\mathrm{H}_{1}\left(K_{g} \cap P_{S} ; \mathbb{Q}\right)$ is spanned by $U_{1} \cup U_{2} \cup U_{3}$

2. If the genus of $S$ is positive, then $C_{g} \cap \mathrm{H}_{1}\left(K_{g} \cap P_{S} ; \mathbb{Q}\right)$ is spanned by $U_{2}$ and $\mathrm{H}_{1}\left(K_{g} \cap P_{S} ; \mathbb{Q}\right)$ is spanned by $U_{1} \cup U_{2}$.

Proof. Define

$$
K_{S}=\operatorname{ker}\left(\pi_{1}(S) \rightarrow \mathrm{H}_{1}(S ; \mathbb{Z} / L)\right) \quad \text { and } \quad C_{S}=\operatorname{ker}\left(\mathrm{H}_{1}\left(K_{S} ; \mathbb{Q}\right) \rightarrow \mathrm{H}_{1}(S ; \mathbb{Q})\right) .
$$

Since $\Sigma_{g} \backslash S$ is connected, the map $\mathrm{H}_{1}(S ; \mathbb{Z} / L) \rightarrow \mathrm{H}_{1}\left(\Sigma_{g} ; \mathbb{Z} / L\right)$ is injective. Using the commutative diagram

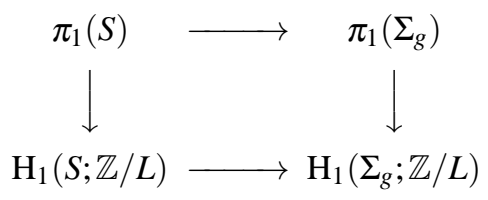

we deduce that the natural maps

$$
K_{S} \longrightarrow K_{g} \cap P_{S} \quad \text { and } \quad C_{S} \longrightarrow C_{g} \cap \mathrm{H}_{1}\left(K_{g} \cap P_{S} ; \mathbb{Q}\right)
$$

are surjective. 
Define

$$
\begin{gathered}
V_{1}^{\prime}=\left\{x^{L} \in K_{S} \mid x \in \pi_{1}(S)\right. \text { can be realized by a simple closed curve that is either } \\
\text { nonseparating or freely homotopic to a boundary component }\},
\end{gathered}
$$

There is a short exact sequence

$$
1 \longrightarrow\left[\pi_{1}(S), \pi_{1}(S)\right] \longrightarrow K_{S} \longrightarrow L \cdot \mathrm{H}_{1}(S ; \mathbb{Z}) \longrightarrow 1
$$

where $L \cdot \mathrm{H}_{1}(S ; \mathbb{Z})$ denotes the subgroup $\left\{L \cdot v \mid v \in \mathrm{H}_{1}(S ; \mathbb{Z})\right\}$. The subgroup of $K_{S}$ generated by $V_{1}^{\prime}$ surjects onto $L \cdot \mathrm{H}_{1}(S ; \mathbb{Z})$. Also, making use of a standard basis for $\pi_{1}(S)$, one can easily check that $V_{2}^{\prime} \cup V_{3}^{\prime}$ generates $\left[\pi_{1}(S), \pi_{1}(S)\right]$. Finally, the proof of [19, Theorem A.3] shows that if the genus of $S$ is positive, then $\left[\pi_{1}(S), \pi_{1}(S)\right]$ is generated by $V_{2}^{\prime}$. The upshot of this is that $K_{S}$ is generated by $V_{1}^{\prime} \cup V_{2}^{\prime} \cup V_{3}^{\prime}$ in all cases and by $V_{1}^{\prime} \cup V_{2}^{\prime}$ if the genus of $S$ is positive.

Defining $V_{i}$ to be the image of $V_{i}^{\prime}$ in $\mathrm{H}_{1}\left(K_{S} ; \mathbb{Q}\right)$, we obtain that $\mathrm{H}_{1}\left(K_{S} ; \mathbb{Q}\right)$ (resp. $\left.C_{S}\right)$ is spanned by $V_{1} \cup V_{2} \cup V_{3}$ (resp. $V_{2} \cup V_{3}$ ) in all cases and by $V_{1} \cup V_{2}$ (resp. $V_{2}$ ) if the genus of $S$ is positive. The set $V_{i}$ maps to $U_{i}$ under the natural map $\mathrm{H}_{1}\left(K_{S} ; \mathbb{Q}\right) \rightarrow \mathrm{H}_{1}\left(K_{g} \cap P_{S} ; \mathbb{Q}\right)$. Since this map and the restricted map $C_{S} \rightarrow C_{g} \cap \mathrm{H}_{1}\left(K_{g} \cap P_{S} ; \mathbb{Q}\right)$ are surjections, the lemma follows

Proof of Lemma 8.1. Since $x$ and $y$ are essentially separate, we can decompose $\Sigma_{g}$ into the union of two connected subsurfaces $S_{1}$ and $S_{2}$ with the following properties.

1. $S_{1}$ and $S_{2}$ both contain the basepoint.

2. $S_{1} \cap S_{2}=\partial S_{1}=\partial S_{2}$.

3. $x \in \operatorname{Im}\left(\pi_{1}\left(S_{1}\right) \rightarrow \pi_{1}\left(\Sigma_{g}\right)\right)$ and $y \in \operatorname{Im}\left(\pi_{1}\left(S_{2}\right) \rightarrow \pi_{1}\left(\Sigma_{g}\right)\right)$.

Applying Lemma 8.2 to each $S_{i}$, we can write

$$
\langle\langle x\rangle\rangle=\sum_{i=1}^{k}\left\langle\left\langle x_{i}\right\rangle\right\rangle^{v_{i}} \quad \text { and } \quad\langle\langle y\rangle\rangle=\sum_{j=1}^{k^{\prime}}\left\langle\left\langle y_{j}\right\rangle\right\rangle^{v_{j}^{\prime}}
$$

where $v_{i}, v_{j}^{\prime} \in H_{L}$ and $x_{i}$ and $y_{j}$ satisfy the following conditions.

- $x_{i} \in \operatorname{Im}\left(\pi_{1}\left(S_{1}\right) \rightarrow \pi_{1}\left(\Sigma_{g}\right)\right)$ and $y_{j} \in \operatorname{Im}\left(\pi_{1}\left(S_{2}\right) \rightarrow \pi_{1}\left(\Sigma_{g}\right)\right)$ for all $i$ and $j$. In particular, the curves $x_{i}$ and $y_{j}$ are essentially separate for all $i$ and $j$.

- $x_{i}$ is either a genus 1 separating curve, the commutator of a nonseparating figure eight, or $z^{L}$ for some simple closed nonseparating curve $z$.

- $y_{j}$ is either a genus 1 separating curve or the commutator of a nonseparating figure eight.

We then have

$$
\langle\langle x\rangle\rangle^{v} \otimes\langle\langle y\rangle\rangle^{v^{\prime}}=\sum_{i=1}^{k} \sum_{j=1}^{k^{\prime}}\left\langle\left\langle x_{i}\right\rangle\right\rangle^{v+v_{i}} \otimes\left\langle\left\langle y_{j}\right\rangle\right\rangle^{v^{\prime}+v_{j}^{\prime}} .
$$

We are almost done - the only problem is that $x_{i}$ or $y_{j}$ might be the commutator of a nonseparating figure eight for some $i$ or $j$. However, if that happens, then we may perform the above procedure again to the pair $\left\{x_{i}, y_{j}\right\}$, but this time it is easy to see that we may ensure that the genera of $S_{1}$ and $S_{2}$ are both positive, in which case we do not need to use nonseparating figure eights. 


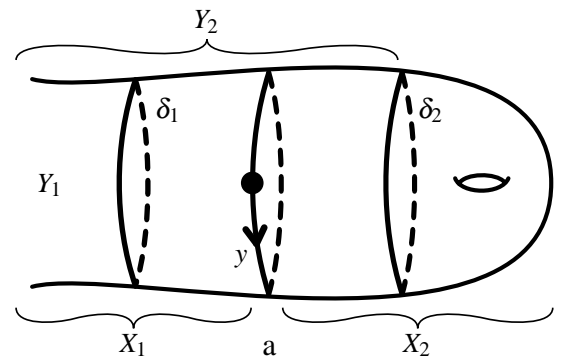

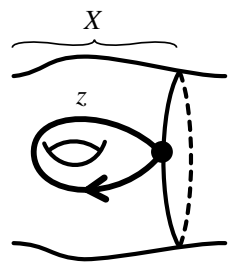

b

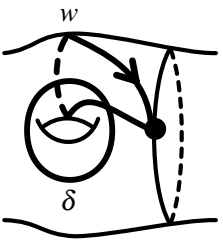

$\mathrm{c}$

Figure 10: $a$. Figure needed to deal with elements of $\mathscr{S}_{g}(1) . \quad b . \quad$ The curve $z$ can be realized by a simple closed nonseparating curve and lies entirely in $X \subset \Sigma_{g} . \quad c . T_{\delta}^{e L}(w)=z^{L} w$. The sign $e= \pm 1$ depends on the orientation of $z$; with the orientation depicted in the figure, we have $e=1$.

\subsection{The proof of Lemma 5.5}

In this final section, we prove Lemma 5.5. We begin by briefly recalling its statement. The set

$$
\mathscr{K}_{g}<\mathrm{H}_{1}\left(K_{g} ; \mathbb{Z}\right) \otimes C_{g}=\mathrm{H}_{1}\left(K_{g} ; C_{g}\right)
$$

is the span of the set

$$
\left\{x \otimes y \mid(x, y) \in \mathscr{S}_{g}\right\} \cup\left\{x \otimes y-f(x) \otimes f(y) \mid x \in \mathrm{H}_{1}\left(K_{g} ; \mathbb{Z}\right), y \in C_{g}, f \in \operatorname{Mod}_{g}^{1}(L)\right\} .
$$

Here $\mathscr{S}_{g}$ is the set defined in $§ 5.2$. Lemma 5.5 asserts that for $g \geq 4$, the image of $\mathscr{K}_{g}$ in $\mathrm{H}_{1}\left(\operatorname{Mod}_{g}^{1}(L) ; C_{g}\right)$ is zero.

Let $\phi: \mathrm{H}_{1}\left(K_{g} ; \mathbb{Z}\right) \otimes C_{g} \rightarrow \mathrm{H}_{1}\left(\operatorname{Mod}_{g}^{1}(L) ; C_{g}\right)$ be the natural map. Since inner automorphisms act trivially on homology, we have

$$
\phi(x \otimes y)=\phi(f(x) \otimes f(y))
$$

for all $x \otimes y \in \mathrm{H}_{1}\left(K_{g} ; \mathbb{Z}\right) \otimes C_{g}$ and $f \in \operatorname{Mod}_{g}^{1}(L)$. We must show that in addition we have $\phi(s)=0$ for $s \in \mathscr{S}_{g}$. This will require several steps.

Step 1. Consider $v, v^{\prime} \in H_{L}$ and $x, y \in K_{g}$ such that $x$ and $y$ are essentially separate and $\langle\langle y\rangle\rangle \in C_{g}$, so $\left(\langle\langle x\rangle\rangle^{v},\langle\langle y\rangle\rangle^{v^{\prime}}\right) \in \mathscr{S}_{g}(1)$. Then $\phi\left(\langle\langle x\rangle\rangle^{v} \otimes\langle\langle y\rangle\rangle^{v^{\prime}}\right)=0$.

By Lemma 8.1, we can assume that one of the following two conditions hold.

1. $\{x, y\}$ has the same unoriented intersection pattern as the curves in Figure 9.a, or

2. $x=\left(x^{\prime}\right)^{L}$, where $\left\{x^{\prime}, y\right\}$ has the same unoriented intersection pattern as the curves in Figure 9.b.

Thus $y$ is a separating curve separating $\Sigma_{g}$ into two subsurfaces $X_{1}$ and $X_{2}$ with $X_{2} \cong \Sigma_{1,1}$ (see Figure 10.a). Also, $x$ is in $\operatorname{Im}\left(\pi_{1}\left(X_{1}\right) \rightarrow \pi_{1}\left(\Sigma_{g}\right)\right)$.

The group $\operatorname{Mod}_{g}^{1}(L)$ contains all inner automorphisms of $\pi_{1}\left(\Sigma_{g}\right)$, so using (14) we can assume that $v=0$. We will produce a subgroup $\Gamma<\operatorname{Mod}_{g}^{1}(L)$ with the following three properties.

1. $\mathrm{H}_{1}(\Gamma ; \mathbb{Q})=0$.

2. $\Gamma$ acts trivially on $\langle\langle y\rangle$.

3. $\Gamma$ contains the "point-pushing" mapping class corresponding to $x \in \pi_{1}\left(\Sigma_{g}\right)$.

This is enough to prove the desired claim. Indeed, since $\Gamma<\operatorname{Mod}_{g}^{1}(L)$, we have $f\left(v^{\prime}\right)=v^{\prime}$ for all $f \in \Gamma$. This and item 2 imply that $\Gamma$ fixes $\langle\langle y\rangle\rangle^{\prime}$. There is thus a map $j: \mathrm{H}_{1}(\Gamma ; \mathbb{Q}) \rightarrow \mathrm{H}_{1}\left(\operatorname{Mod}_{g}^{1}(L) ; C_{g}\right)$ corresponding to the inclusion $\Gamma \hookrightarrow \operatorname{Mod}_{g}^{1}(L)$ and the map $\mathbb{Q} \hookrightarrow C_{g}$ taking $1 \in \mathbb{Q}$ to $\langle\langle y\rangle\rangle^{\nu^{\prime}}$. By item 3 , the image of $j$ contains $\phi\left(\langle\langle x\rangle\rangle^{v} \otimes\langle\langle y\rangle\rangle^{v^{\prime}}\right)$, so by item 1 we have $\phi\left(\langle\langle x\rangle\rangle^{v} \otimes\langle\langle y\rangle\rangle^{\prime}\right)=0$, as desired. 
We construct $\Gamma$ as follows. Let $N$ be a regular neighborhood of $y$ and let $\delta_{i}$ be the boundary component of $N$ that is contained in $X_{i}$ (see Figure 10.a). The curve $\delta_{i}$ separates $\Sigma_{g}$ into two components. Let $Y_{i}$ be the component of $\Sigma_{g}$ cut along $\delta_{i}$ that intersects $X_{1}$. It follows that $Y_{1} \cong \Sigma_{g-1,1}$ and $Y_{2} \cong \Sigma_{g-1,1}^{1}$. Denoting the mapping class group of $Y_{i}$ by $\operatorname{Mod}\left(Y_{i}\right)$, we have a Birman exact sequence

$$
1 \longrightarrow \pi_{1}\left(\Sigma_{g-1,1}\right) \longrightarrow \operatorname{Mod}\left(Y_{2}\right) \longrightarrow \operatorname{Mod}_{g-1,1} \longrightarrow 1 .
$$

We have an isomorphism $\operatorname{Mod}_{g-1,1} \cong \operatorname{Mod}\left(Y_{1}\right)$, and this exact sequence splits via a map that identifies $f \in$ $\operatorname{Mod}_{g-1,1}$ with a corresponding element of $\operatorname{Mod}\left(Y_{1}\right)$ and then extends $f$ by the identity to an element of $\operatorname{Mod}\left(Y_{2}\right)$. Choosing such a splitting, we get a decomposition

$$
\operatorname{Mod}\left(Y_{2}\right)=\pi_{1}\left(\Sigma_{g-1,1}\right) \rtimes \operatorname{Mod}_{g-1,1} .
$$

Define

$$
\Gamma=K_{g-1,1} \rtimes \operatorname{Mod}_{g-1,1}(L)<\pi_{1}\left(\Sigma_{g-1,1}\right) \rtimes \operatorname{Mod}_{g-1,1} .
$$

It is clear that $\Gamma$ contains the "point-pushing" mapping class corresponding to $x \in \pi_{1}\left(\Sigma_{g}\right)$. Also, the subgroup $\operatorname{Mod}_{g-1,1}(L)<\Gamma$ fixes $y \in \pi_{1}\left(\Sigma_{g}\right)$ and the subgroup $K_{g-1,1}<\Gamma$ acts on $y$ by conjugation, and thus fixes $\langle y y\rangle$. We deduce that $\Gamma$ acts trivially on $\langle\langle y\rangle\rangle$.

It remains to check that $\mathrm{H}_{1}(\Gamma ; \mathbb{Q})=0$. From its semidirect product decomposition, we deduce that

$$
\mathrm{H}_{1}(\Gamma ; \mathbb{Q}) \cong \mathrm{H}_{1}\left(\operatorname{Mod}_{g-1,1}(L) ; \mathbb{Q}\right) \oplus\left(\mathrm{H}_{1}\left(K_{g-1,1} ; \mathbb{Q}\right)\right)_{\operatorname{Mod}_{g-1,1}(L)} .
$$

Since $g \geq 4$, Theorem 2.6 implies that $\mathrm{H}_{1}\left(\operatorname{Mod}_{g-1,1}(L) ; \mathbb{Q}\right)=0$. Again using the fact that $g \geq 4$, Lemma 3.4 implies that $\left(\mathrm{H}_{1}\left(K_{g-1,1} ; \mathbb{Q}\right)\right)_{\operatorname{Mod}_{g-1,1}(L)}=0$, and we are done.

Step 2. $\phi(a \otimes b)=0$ if $(a, b) \in \mathscr{S}_{g}(2)$.

Consider $\left(\langle\langle x\rangle\rangle^{\nu},\left\langle\left\langle y, z^{L}\right\rangle\right\rangle^{\prime}\right) \in \mathscr{S}_{g}(2)$, so $z$ can be realized by a simple closed nonseparating curve and $\{z\}$ and $\{x, y\}$ are strongly essentially disjoint. We can thus find subsurfaces $X$ and $X^{\prime}$ of $\Sigma_{g}$ both of which contain the basepoint such that

$$
z \in \operatorname{Im}\left(\pi_{1}(X) \rightarrow \pi_{1}\left(\Sigma_{g}\right)\right) \quad \text { and } \quad\{x, y\} \subset \operatorname{Im}\left(\pi_{1}\left(X^{\prime}\right) \rightarrow \pi_{1}\left(\Sigma_{g}\right)\right) .
$$

and such that

$$
\Sigma_{g}=X \cup X^{\prime} \quad \text { and } \quad X \cap X^{\prime}=\partial X=\partial X^{\prime} .
$$

Additionally, the surfaces $X$ and $X^{\prime}$ can be chosen such that both have only one boundary component.

As is shown in Figure 10.c, there exists some $w \in \operatorname{Im}\left(\pi_{1}(X) \rightarrow \pi_{1}\left(\Sigma_{g}\right)\right)$ together with an unbased simple closed curve $\delta$ in $X \subset \Sigma_{g}$ such that $T_{\delta}^{e L}(w)=z^{L} w$ for some $e= \pm 1$. Since $\delta \subset X$, we have $T_{\delta}(x)=x$ and $T_{\delta}(y)=z$. Also, since $T_{\delta}^{e L} \in \operatorname{Mod}_{g}^{1}(L)$, it follows that $T_{\delta}^{e L}$ acts trivially on $v$ and $v^{\prime}$. This implies that $T_{\delta}^{e L}$ takes $\langle\langle x\rangle\rangle^{v} \otimes\langle\langle y, w\rangle\rangle^{v^{\prime}}$ to

$$
\begin{aligned}
\langle\langle x\rangle\rangle^{v} \otimes\left\langle\left\langle y, z^{L} w\right\rangle\right\rangle v^{v^{\prime}} & =\langle\langle x\rangle\rangle^{v} \otimes\left\langle\left\langle y, z^{L}\right\rangle\right\rangle^{v^{\prime}}+\langle\langle x\rangle\rangle^{v} \otimes\langle\langle y, w\rangle\rangle v^{v^{\prime}+L \cdot \bar{z}} \\
& =\langle\langle x\rangle\rangle^{v} \otimes\left\langle\left\langle y, z^{L}\right\rangle\right\rangle^{v^{\prime}}+\langle\langle x\rangle\rangle^{v} \otimes\langle\langle y, w\rangle\rangle^{v^{\prime}} .
\end{aligned}
$$

The first calculation here uses Lemma 5.1 and the second the fact that $L \cdot \bar{z}=0$. Since $\operatorname{Mod}_{g}^{1}(L)$ acts trivially on the image of $\phi$, we conclude that

$$
\phi\left(\langle\langle x\rangle\rangle^{v} \otimes\langle\langle y, w\rangle\rangle^{\nu^{\prime}}\right)=\phi\left(\langle\langle x\rangle\rangle^{v} \otimes\langle\langle y, w\rangle\rangle^{v^{\prime}}+\langle\langle x\rangle\rangle^{v} \otimes\left\langle\left\langle y, z^{L}\right\rangle\right\rangle^{\nu^{\prime}}\right) ;
$$

i.e. that $\phi\left(\langle\langle x\rangle\rangle^{\nu} \otimes\left\langle\left\langle y, z^{L}\right\rangle\right\rangle^{\prime}\right)=0$, as desired. 


\section{References}

[1] J. Anderson and R. Villemoes, Degree one cohomology with twisted coefficients of the mapping class group, preprint 2007.

[2] J. Anderson and R. Villemoes, The first cohomology of the mapping class group with coefficients in algebraic functions on the $\mathrm{SL}_{2}(\mathbb{C})$ moduli space, Algebr. Geom. Topol. 9 (2009), 1177-1199.

[3] J. Anderson and R. Villemoes, Cohomology of mapping class groups and the abelian moduli space, preprint 2009.

[4] J. S. Birman, Braids, links, and mapping class groups, Ann. of Math. Stud., 82, Princeton Univ. Press, Princeton, N.J., 1974.

[5] S. Boldsen, Improved homological stability for the mapping class group with integral or twisted coefficients, preprint 2009.

[6] K. S. Brown, Cohomology of groups, Corrected reprint of the 1982 original, Springer, New York, 1994.

[7] T. Church and A. Pixton, Separating twists and the Magnus representation of the Torelli group, to appear in Geom. Dedicata.

[8] B. Farb and D. Margalit, A primer on mapping class groups, preprint 2010.

[9] R. M. Hain, Torelli groups and geometry of moduli spaces of curves, in Current topics in complex algebraic geometry (Berkeley, CA, 1992/93), 97-143, Cambridge Univ. Press, Cambridge.

[10] J. L. Harer, Stability of the homology of the mapping class groups of orientable surfaces, Ann. of Math. (2) 121 (1985), no. 2, 215-249.

[11] J. Hempel, Intersection calculus on surfaces with applications to 3-manifolds, Mem. Amer. Math. Soc. 43 (1983), no. 282 , vi+48 pp.

[12] N. V. Ivanov, On the homology stability for Teichmüller modular groups: closed surfaces and twisted coefficients, in Mapping class groups and moduli spaces of Riemann surfaces (Göttingen, 1991/Seattle, WA, 1991), 149-194, Contemp. Math., 150, Amer. Math. Soc., Providence, RI.

[13] D. Johnson, The structure of the Torelli group. I. A finite set of generators for $\mathscr{I}$, Ann. of Math. (2) 118 (1983), no. 3, 423-442.

[14] N. Kawazumi, On the stable cohomology algebra of extended mapping class groups for surfaces, in Groups of Diffeomorphisms, 383-400, Adv. Stud. Pure Math., 52, Math. Soc. Japan, Tokyo.

[15] E. Looijenga, Stable cohomology of the mapping class group with symplectic coefficients and of the universal Abel-Jacobi map, J. Algebraic Geom. 5 (1996), no. 1, 135-150.

[16] E. Looijenga, Prym representations of mapping class groups, Geom. Dedicata 64 (1997), no. 1, 69-83.

[17] I. Madsen and M. Weiss, The stable moduli space of Riemann surfaces: Mumford's conjecture, Ann. of Math. (2) 165 (2007), no. 3, 843-941.

[18] S. Morita, Families of Jacobian manifolds and characteristic classes of surface bundles. I, Ann. Inst. Fourier (Grenoble) 39 (1989), no. 3, 777-810.

[19] A. Putman, Cutting and pasting in the Torelli group, Geom. Topol. 11 (2007), 829-865.

[20] A. Putman, The second rational homology group of the moduli space of curves with level structures, preprint 2008.

[21] A. Putman, The Picard group of the moduli space of curves with level structures, preprint 2009.

[22] K. Reidemeister, Homotopiegruppen von Komplexen, Abh. Math. Sem. Hamburgischen Univ. 10 (1935), $211-215$.

[23] K. Reidemeister, Complexes and homotopy chains, Bull. Amer. Math. Soc. 56 (1950), 297-307.

Andrew Putman

Department of Mathematics

Rice University, MS 136

6100 Main St.

Houston, TX 77005

E-mail: andyp@rice.edu 